Article

\title{
Copper Mineralization Potential of Late Triassic Granitoids in Northern Yidun Arc, SW China
}

\author{
Xing-Yuan Li ${ }^{1,2, *}$, Jing-Ru Zhang ${ }^{3}$, Hao Song ${ }^{2, *}$ and Chun-Kit Lai ${ }^{4,5}$ (D) \\ 1 School of Geography and Planning, Sun Yat-sen University, Guangzhou 510275, China \\ Department of Geology, University of Regina, Regina, SK S4S 0A2, Canada \\ Guangdong Province Academic of Environmental Science, Guangzhou 510045, China; \\ zhangjrgucas@163.com \\ 4 Faculty of Science, Universiti Brunei Darussalam, Gadong BE1410, Brunei; chunkit.lai@ubd.edu.bn \\ 5 Centre of Excellence in Ore Deposits (CODES), University of Tasmania, Tasmania 7001, Australia \\ * Correspondence: lixingyuan@mail.sysu.edu.cn (X.-Y.L.); songhao2014@cdut.edu.cn (H.S.); \\ Tel.: +86-020-8411-2307 (X.-Y.L.)
}

Received: 9 May 2019; Accepted: 27 May 2019; Published: 1 June 2019

\begin{abstract}
Yidun arc is an important constituent of the Sanjiang Tethyan Domain in SW China. The Changdagou pluton, located in the northern part of the Yidun Arc, mainly consists of granodiorite. In this study, we conducted in-situ LA-ICP-MS zircon U-Pb dating, and trace element and Hf isotope analyses on the Changdagou granites. Age dating results yielded a weighted mean $\mathrm{U}-\mathrm{Pb}$ age of $214.97 \pm 0.98 \mathrm{Ma}(\mathrm{MSWD}=1.2,2 \sigma)$, broadly coeval with extensive late Triassic magmatism across the Yidun Arc. All zircon grains analyzed showed high concentrations of Th, U, and HREE, with positive $\mathrm{Ce}$ and negative Eu anomalies. $\log f o_{2}$ and $\mathrm{Ce}_{\mathrm{N}} / \mathrm{Ce}_{\mathrm{N}}{ }^{*}$ values vary from FMQ -3.14 to FMQ +7.44 (average FMQ +3.98), and 14 to 172 (avg. 98), respectively. The zircon $\mathrm{Eu}_{\mathrm{N}} / \mathrm{Eu}_{\mathrm{N}}{ }^{*}$ (avg. 0.22) ratios have no clear correlation with the $\mathrm{Ce}_{\mathrm{N}} / \mathrm{Ce}_{\mathrm{N}}{ }^{*}$ ratios, suggesting that the former were mainly affected by the magma water content. In addition, zircon $\varepsilon \operatorname{Hf}(t)$ values vary in a narrow range $(-2.9$ to -4.9 , avg. -3.4$)$ that clusters around zero, indicating a greater component of mantle-derived magma. Hence, we propose that the Changdagou granodiorite was derived from a highly oxidized, "wet", $\mathrm{Cu}$-rich source, of the type likely to generate porphyry $\mathrm{Cu}$ mineralization. However, these parameters $\left(\log f \mathrm{O}_{2}, \mathrm{Eu}_{\mathrm{N}} / \mathrm{Eu}_{\mathrm{N}}{ }^{*},(\mathrm{Ce} / \mathrm{Nd}) / \mathrm{Y}\right.$, and $\left.\varepsilon \mathrm{Hf}(\mathrm{t})\right)$ are all lower than those of intrusions associated with $\mathrm{Cu}$ ores at Pulang and Lannitang, which may explain why the $\mathrm{Cu}$ deposit discovered at Changdagou is small by comparison. Furthermore, on the basis of the decreasing trends of $\varepsilon \mathrm{Hf}, \log f \mathrm{O}_{2}$, and $\mathrm{H}_{2} \mathrm{O}$ content from south to north along the Yiduan arc, we infer that the northern segment of the Yidun arc (including Changdagou) was located further away from the subduction front.
\end{abstract}

Keywords: Yidun arc; Changdagou granite; porphyry Cu deposits; paleotethys; zircon chemistry and isotopes

\section{Introduction}

The Sanjiang Tethyan Domain in the southeastern Tibet Plateau comprises various (micro)-continental blocks, volcanic arcs, and ophiolitic belts that were formed during the opening and closure of the Paleo-Tethys (Figure 1) [1]. The NNW-trending Yidun structure is the largest island arc preserved in the domain [2-4], and a number of important porphyry copper deposits (PCDs) were found in its southern section, such as the Pulang, Lannitang and Xuejiping. These PCDs were mainly formed in the Late Triassic (208-235 Ma) during the Indosinian Orogeny [5-7]. In contrast, only a few PCDs have been discovered in the northern section of the arc, with the Changdagou PCD being the largest one [8]. This raises questions on whether the paucity of PCD discovery is an issue of exploration work, deposit preservation, or a combination of these factors. 


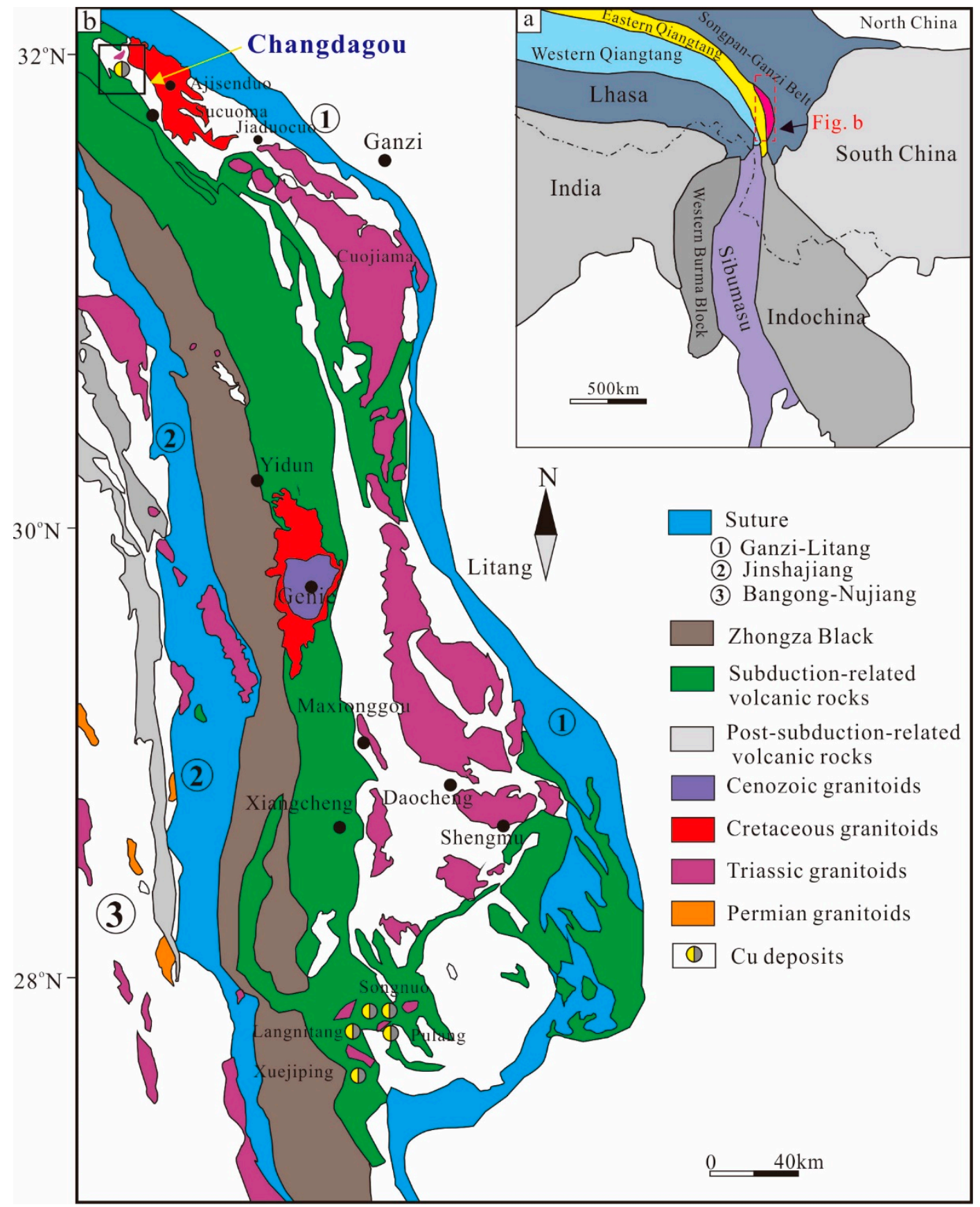

Figure 1. (a) Tectonic framework and distribution of major geological terranes and sutures of mainland SE Asia; (b) Tectonic framework of the Sanjiang Region, including the major geological terranes, sutures, volcanic rocks and intrusions (modified after [6]).

Redox state and water content of magmas are widely believed to be contributing factors for the formation of PCDs. Recent studies [9-14] revealed that zircon grain composition is sensitive to these factors, and can serve as an indicator of the magma physicochemical conditions. Ballard et al. [15] suggest PCDs are associated with zircon $\mathrm{Ce}^{4+} / \mathrm{Ce}^{3+}>300$ of magma, whilst Dilles [12] suggest that PCD fertile magmas commonly have $\mathrm{Eu}_{\mathrm{N}} / \mathrm{Eu}_{\mathrm{N}}{ }^{*}>0.3$. More recently, Lu et al. [14] argue that zircon trace element ratios, such as $\mathrm{Eu}_{N} / \mathrm{Eu}_{\mathrm{N}}{ }^{*}, \mathrm{Dy} / \mathrm{Yb}$, and $\left(\mathrm{Eu}_{\mathrm{N}} / \mathrm{Eu}_{\mathrm{N}}{ }^{*}\right) / \mathrm{Y}$, can serve as proxies for the water content in magma. In addition, Hf isotopic signatures $(\varepsilon H f)$ can indicate whether the magma source was juvenile (with higher $\varepsilon \mathrm{Hf}$, i.e., close to the mantle value and enriched in radiogenic ${ }^{176} \mathrm{Hf}$ ) or from an evolved, typically ancient crustal source (with lower $\varepsilon$ Hf; [16-18]). 
In this paper, we present a new zircon $\mathrm{U}-\mathrm{Pb}$ age, trace element chemical and $\mathrm{Hf}$ isotope data on the Changdagou granodiorite porphyry in the northern Yidun arc. We discuss the redox state, water content, and possible magmatic source of the Changdagou granites, and compare these parameters with those in the Lannitang and Pulang granites in the southern Yidun arc. We evaluate some differences in mineralization potential between the northern and southern sections of the Yidun arc.

\section{Geological Background}

The Yidun arc and its PCDs have been described in many publications $[1,16,19]$. In brief, the Yidun arc is located between the Jinshajiang and Ganzi-Litang Paleo-Tethyan suture zones [2,20,21]. During the Late Triassic, closure of the Ganzi-Litang ocean basin might have led to the accretion of the Zhongza terrane onto the Yangtze craton via the Yidun arc [22] (Figure 1). It has been suggested that the Yidun arc was developed on the basement with the Yangtze craton inheritance [4,6,19], and underwent two magmatic episodes in the Late Triassic (218-230 Ma) and Late Cretaceous (88-105 Ma), responding the Paleo-Tethyan subduction and subsequent continent-continent collision (Figure 1a). The subduction-related magmatism formed several large granodioritic-granitic batholiths (e.g., Changdagou, Shengmu, Cuojiaoma and Daocheng) in the northern Yidun arc [16,22], and dioritic-granitic stocks (e.g., Pulang and Lannitang) in the southern Yidun arc (also called the Zhongdian arc) [11,23-25].

The Changdagou PCD ( $\sim 35 \mathrm{~km}$ from Dege city) is located in the northern Yidun arc, and contains $\sim 76 \mathrm{kt} \mathrm{Cu}$ reserve [8]. The Changdagou intrusive complex intruded the black-gray slate and sandstone of the Upper Triassic Lanashan Formation (Figure 2). The intrusive complex comprises mainly granodiorite porphyry and minor quartz diorite porphyry stocks, and Changdagou PCD is closely related to the granodiorite porphyry. In this study, one granodiorite porphyry sample was collected from drill cores, which contain quartz (10-15\%), plagioclase (25-55\%), K-feldspar (10-30\%) and biotite $(3-5 \%)$, with accessory titanite, apatite, pyrite, and magnetite.

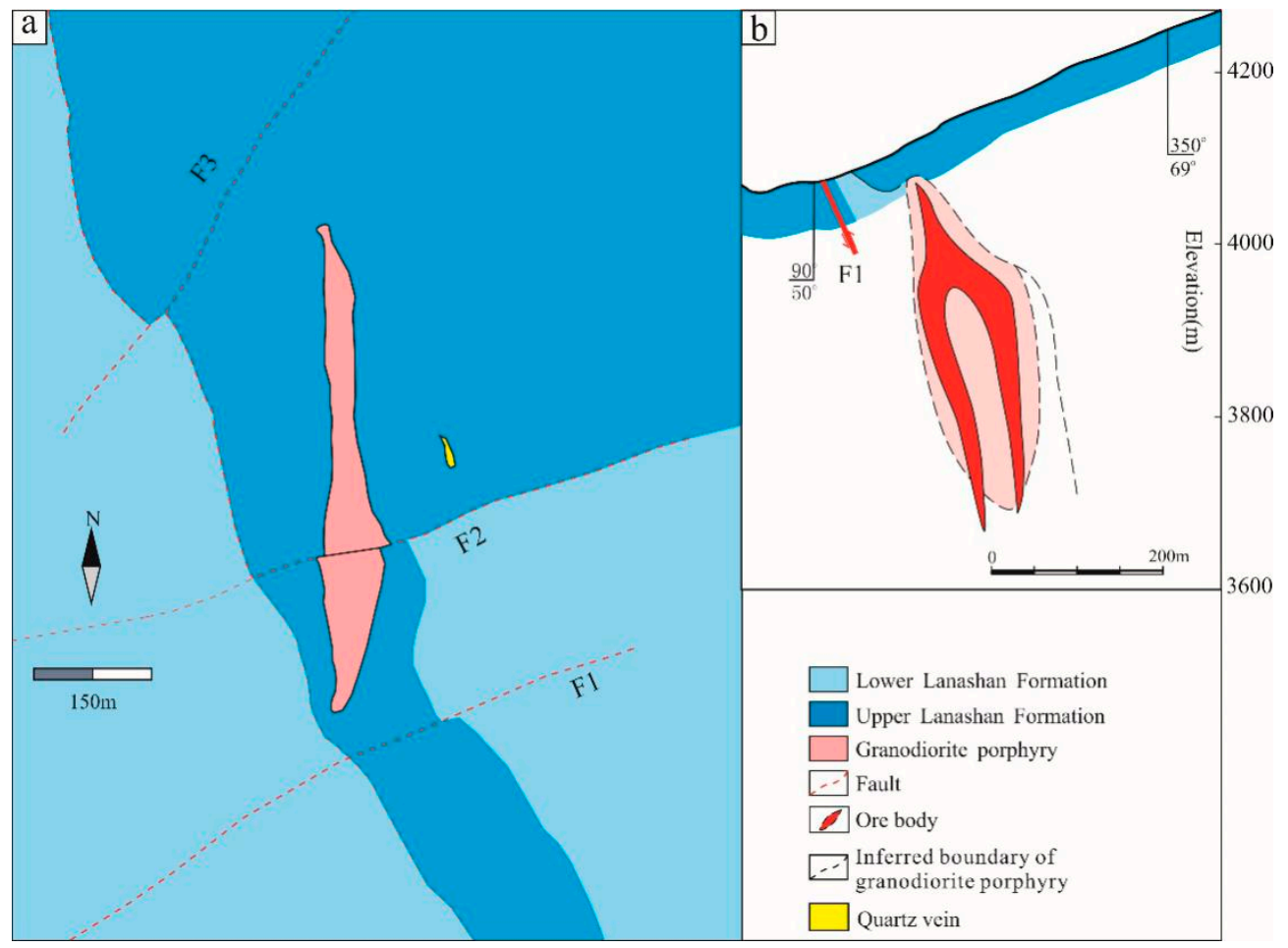

Figure 2. (a) Simplified geologic map of the Changdagou deposit (modified from [6]). (b) Geological profile for prospecting line No. 12. 
Major alteration types in the Changdagou granite are silicic, potassic, sericite, argillic (kaolinite) and propylitic. Silicification occurs mainly in the ore-forming granodiorite and the silicified rocks in its exo-contact, and the orebody in the silicified zone is of higher grade. Quartz-calcite veins intersect each other in a grid pattern, and are unevenly distributed in the intrusion. Potassic alteration is distinct, and often contains veinlet/disseminated sulfide mineralization. Sericitization is most prevalent in the mining area, and is manifested as fine scaly aggregates in the metasomatic matrix. Mineralization intensity at Changdagou is clearly related to the alteration style. Strongly mineralized and highest grade (up to $1.64 \%$ ) areas are mainly concentrated in the potassic and silicic zones. Low-grade mineralization $(<0.4 \%)$ is also developed in/around the sericite zones.

\section{Methods}

\subsection{Zircon Morphology and Texture}

Zircon separation was conducted on $\sim 2 \mathrm{~kg}$ crushed rock sample at the Langfang Geological Laboratory (Langfang, China). Approximately 100-200 zircon grains from each sample were mounted in an epoxy resin disc. Before the U-Pb isotope analysis, all grains were observed under transmitted and reflected-light microscopy, as well as cathodoluminescence (CL) imaging to reveal their internal structure. All the CL imaging, U-Pb dating, trace element chemical and Hf isotopic analyses were performed at the Wuhan Sample Solution Analytical Technology Co. Ltd. (Wuhan, China). Zircon CL imaging was conducted with the Analytical Scanning Electron Microscope (JSMIT100, JEOL, Tokyo, Japan) connected to a GATAN MINICL system. The imaging conditions include 10,013.0 kV electric field and $8085 \mu \mathrm{A}$ current of tungsten filament.

\subsection{Zircon U-Pb Dating and Trace-Element Analyses}

$\mathrm{U}-\mathrm{Pb}$ dating and trace element analyses on zircon were simultaneously conducted with laser ablation-inductively coupled plasma-mass spectrometry (LA-ICP-MS). Detailed operating conditions for the laser ablation system and the ICP-MS instrument and data reduction are as described by Zong et al. [26]. The analyses were performed with a GeolasPro laser ablation system that consists of a COMPexPro 102 ArF excimer laser (193 nm wavelength and $200 \mathrm{~mJ}$ maximum energy) and a MicroLas optical system. An Agilent 7700e ICP-MS instrument (Agilent Technologies, Santa Clara, CA, USA) was used to acquire ion-signal intensities. Helium was used as a carrier gas, and argon was used as the make-up gas that mixed with the carrier gas via a T-connector before entering the ICP. A "wire" signal smoothing device is included in this laser ablation system [27]. The spot size and frequency of the laser were set to $32 \mu \mathrm{m}$ and $5 \mathrm{~Hz}$, respectively. Zircon GJ-1 was used as the external standards for U-Pb dating and trace element calibration [28], respectively. Each analysis consists of a $20-30$ s background acquisition followed by a $50 \mathrm{~s}$ sample data acquisition. In addition, the off-line selection and integration of background and analyzed signals, quantitative calibration for trace element analysis and time-drift correction and U-Pb dating were carried out with the Excel-based ICPMSDataCal software (Version 10.9, China University of Gersciences, Wuhan, China) [27]. The common lead correction was corrected with the measured ${ }^{204} \mathrm{~Pb}$ concentration [28,29]. Concordia diagrams and weighted mean calculations were calculated by the Isoplot/Ex_ver3 [30].

\subsection{Zircon Hf Isotopic Analyses}

The in-situ zircon Hf analyses were carried out by the Neptune multicollector (Thermo Fisher Scientific, Waltham, MA, USA) inductively coupled plasma-mass spectrometry (MC-ICP-MS) instrument equipped with a Geolas-193 laser ablation system at the Wuhan Sample Solution Analytical Technology Co. Ltd., Wuhan, China. During the analyses, a spot size of $44 \mu \mathrm{m}$ and laser repetition of $8 \mathrm{~Hz}$ with energy density of $5.3 \mathrm{~J} / \mathrm{cm}^{2}$ were used. Detailed operating conditions and procedures for the MC-ICP-MS analyses are as described in Hu et al. [27]. Notably, the spot locations of Hf isotopic analyses were presented in Figure 3. In addition, in-situ zircon Hf isotopes analysis requires 
careful correction for isobaric interferences on ${ }^{176} \mathrm{Hf}$ (e.g., ${ }^{176} \mathrm{Yb}$ and $\left.{ }^{176} \mathrm{Lu}\right)$. It has been observed that the mass fractionation of $\mathrm{Yb}\left(\beta_{\mathrm{Yb}}\right)$ is not constant over time. Besides, the $\beta_{\mathrm{Yb}}$ obtained from the introduction of solutions is unsuitable for in situ zircon measurements [31]. The miscalculation of the $\beta_{\mathrm{Yb}}$ value will evidently affect the results of the ${ }^{176} \mathrm{Hf} /{ }^{177} \mathrm{Hf}$ ratio. In this research, we used the directly obtained $\beta_{\mathrm{Yb}}$ value from the zircon grains in real-time. Moreover, the ${ }^{173} \mathrm{Yb} /{ }^{171} \mathrm{Yb}$ and ${ }^{179} \mathrm{Hf} /{ }^{177} \mathrm{Hf}$ values were applied to estimate the mass bias of $\mathrm{Yb}\left(\beta_{\mathrm{Yb}}\right)$ and $\mathrm{Hf}\left(\beta_{\mathrm{Hf}}\right)$, which were normalized to ${ }^{173} \mathrm{Yb} /{ }^{171} \mathrm{Yb}=1.13268$ and ${ }^{179} \mathrm{Hf} /{ }^{177} \mathrm{Hf}=0.73255$ [32] with an exponential correction for mass bias. Additionally, interference of ${ }^{176} \mathrm{Yb}$ on ${ }^{176} \mathrm{Hf}$ was corrected by measuring the interference-free ${ }^{173} \mathrm{Yb}$ isotope and utilizing ${ }^{176} \mathrm{Yb} /{ }^{173} \mathrm{Yb}=0.79639$ [32] to calculate ${ }^{176} \mathrm{Yb} /{ }^{177} \mathrm{Hf}$. Similarly, the relatively minor interference of ${ }^{176} \mathrm{Lu}$ on ${ }^{176} \mathrm{Hf}$ was corrected by measuring the intensity of the interference-free ${ }^{175} \mathrm{Lu}$ isotope and using the ${ }^{176} \mathrm{Lu} /{ }^{175} \mathrm{Lu}=0.02656$ [33] to estimate the ${ }^{176} \mathrm{Lu} /{ }^{177} \mathrm{Hf}$ value. Because $\mathrm{Yb}$ and Lu have similar physicochemical properties, the $\beta_{\mathrm{Yb}}$ value was applied to calculate the mass fractionation of $\mathrm{Lu}$. The off-line processing of analytical data (including mass bias calibrations, selection of samples, and blank signals) were performed by software ICPMSDataCal [27].

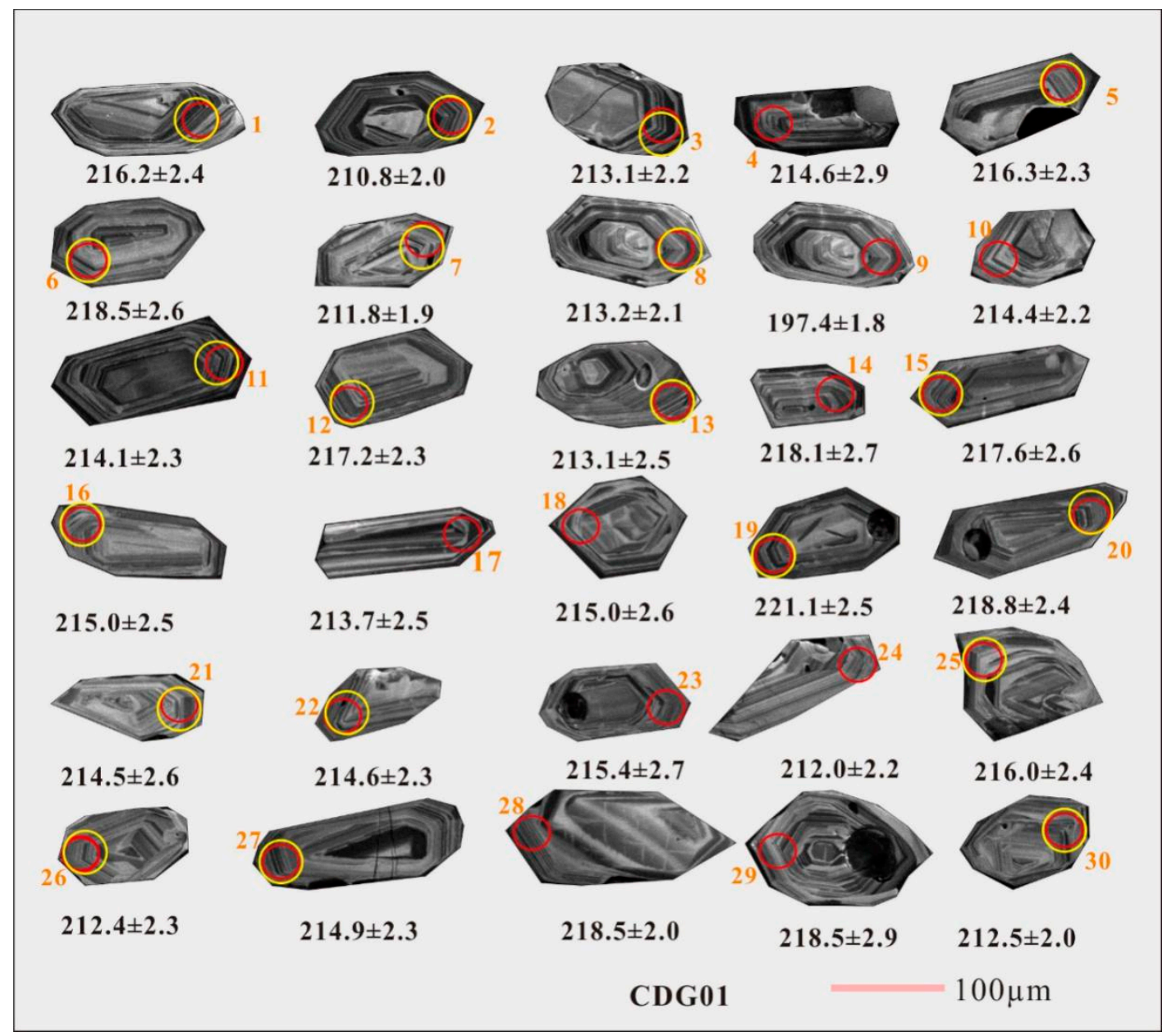

Figure 3. Cathodoluminescence (CL) images of zircons from the Changdagou granodiorite porphyry (sample CDG-01). The larger yellow circles represent locations of Hf isotopic analyses, whereas the smaller red circles indicate spots of LA-ICP-MS U-Pb dating. LA-ICP-MS = laser ablation-inductively coupled plasma-mass spectrometry. 


\subsection{Zircon $\log \mathrm{fO}_{2}$ Estimation}

Chemical compositions of zircon have been used to estimate the magma oxygen fugacity [34-37]. The rare earth element (REE) Ce has two valence states $\left(\mathrm{Ce}^{3+}\right.$ and $\left.\mathrm{Ce}^{4+}\right)$ in magmas with different partition coefficients, and $\mathrm{Ce}^{4+}$ preferentially substitutes $\mathrm{Zr}^{4+}$ in zircon. Equation (1) was derived by ref. [34] to estimate the magma $\log f_{2}$ on the basis of the relationship between Ce concentration and the Ti in zircon temperature (evaluated by the equation of Ferry and Watson [37]), estimated through high P-T experiments [38,39]. In addition, Loader et al. [40] argued that $\mathrm{Ce}_{\mathrm{N}} / \mathrm{Ce}_{\mathrm{N}}{ }^{*}$ (on the basis of the Nd-Sm method in zircon) is unlikely to be affected by mineral inclusions and is thus reliable. Hence, we calculated both $\mathrm{Ce}_{\mathrm{N}} / \mathrm{Ce}_{\mathrm{N}}{ }^{*}$ and $\log f \mathrm{O}_{2}$ from the zircon compositions to unravel the magmatic redox state. $\mathrm{Eu}_{\mathrm{N}}{ }^{*}$ in this study is interpolated from the neighboring elements $\mathrm{Sm}$ and $\mathrm{Gd}$, and is equal to $\left(\mathrm{Sm}_{\mathrm{N}} \times \mathrm{Gd}_{\mathrm{N}}\right)^{1 / 2}$.

$$
\operatorname{Ln}\left(\frac{C e}{C e^{*}}\right)_{D}=(0.1156 \pm 0.0050) \times \operatorname{Ln}\left(f \mathrm{O}_{2}\right)+\frac{13,860 \pm 708}{T(\mathrm{~K})}-6.125 \pm 0.484
$$

where $\left(\mathrm{Ce} / \mathrm{Ce}^{*}\right)_{\mathrm{D}}$ is the Ce anomaly in zircon grain, estimated from the partition coefficients, $\mathrm{T}$ is the absolute temperature of zircon crystallization.

\subsection{Water Content Estimation}

Currently, there are only a few methods for estimating magmatic $\mathrm{H}_{2} \mathrm{O}$ content, including the use of plagioclase, hornblende, zircon hygrometer and melt inclusion analysis [38-44]. In addition, some whole-rock element parameters, like $\mathrm{Sr} / \mathrm{Y}$ and $\delta$ Eu values, can provide estimation of water content in PCD-forming magma [11,43,45]. Lu et al. [14] argued that zircon trace element ratios (e.g., $\mathrm{Eu}_{\mathrm{N}} / \mathrm{Eu}_{\mathrm{N}}{ }^{*}$, $\mathrm{Dy} / \mathrm{Yb},(\mathrm{Ce} / \mathrm{Nd}) / \mathrm{Y}$, and $\left.\left(\mathrm{Eu}_{\mathrm{N}} / \mathrm{Eu}_{\mathrm{N}^{*}}\right) / \mathrm{Y}\right)$ can serve as proxies for magmatic water content, and that PCD-fertile magmas commonly contain low $\mathrm{Dy} / \mathrm{Yb}(<0.3)$ and high $\mathrm{Eu}_{\mathrm{N}} / \mathrm{Eu}_{\mathrm{N}}{ }^{*}(>0.3)$ ratios. It was interpreted that "wet" magmas can suppress early plagioclase crystallization, which causes the low $\mathrm{Dy} / \mathrm{Yb}$ and high $\mathrm{Eu}_{\mathrm{N}} / \mathrm{Eu}_{\mathrm{N}}{ }^{*}$ ratios. However, the $\mathrm{Eu}_{\mathrm{N}} / \mathrm{Eu}_{\mathrm{N}}{ }^{*}$ ratios in zircon not only vary with water content but also with the redox state [14,34,45-47]. Hence, in this study, $(\mathrm{Ce} / \mathrm{Nd}) / \mathrm{Y}$ and $\mathrm{Eu}_{\mathrm{N}} / \mathrm{Eu}_{\mathrm{N}}{ }^{*}$ ratios are both selected as proxies for the water content of magma.

\section{Results}

\subsection{Zircon $\mathrm{U}-\mathrm{Pb}$ Age}

For the zircon grains from Changdagou, CL imaging shows distinct concentric oscillatory zoning in most grains with no inherited cores. The $\mathrm{U}-\mathrm{Pb}$ dating results are listed in Table 1 and illustrated in Figure 4 . The $\mathrm{Th} / \mathrm{U}$ ratios range from 0.32 to 1.14 , distinctly higher than those of typical metamorphic zircons but resembling typical magmatic zircons [48,49]. The zircon grains yielded a weighted mean ${ }^{206} \mathrm{~Pb} /{ }^{238} \mathrm{U}$ age of $214.97 \pm 0.98 \mathrm{Ma}(\mathrm{MSWD}=1.2, n=30$ ). The U-Pb ages of cores and rims of the Changdagou zircon grains are identical [50]. 
Table 1. U-Pb isotopic compositions and ages of zircon grains from Changdagou granodiorite porphyry.

\begin{tabular}{|c|c|c|c|c|c|c|c|c|c|c|c|}
\hline \multicolumn{4}{|c|}{ Element Composition (ppm) } & \multicolumn{4}{|c|}{ Isotopic Element Ratio } & \multicolumn{4}{|c|}{ Isotopic Ages (Ma) } \\
\hline \multirow[b]{2}{*}{ Samples } & \multirow[t]{2}{*}{ Th } & \multirow[t]{2}{*}{$\mathbf{U}$} & \multirow[t]{2}{*}{$\mathrm{Th} / \mathrm{U}$} & \multicolumn{2}{|c|}{${ }^{207} \mathrm{~Pb} /{ }^{235} \mathrm{U}$} & \multicolumn{2}{|c|}{${ }^{206} \mathrm{~Pb} /{ }^{238} \mathrm{U}$} & \multicolumn{2}{|c|}{${ }^{207} \mathrm{~Pb} /{ }^{235} \mathrm{U}$} & \multicolumn{2}{|c|}{${ }^{206} \mathrm{~Pb} /{ }^{238} \mathrm{U}$} \\
\hline & & & & Ratio & $1 \sigma$ & Ratio & $1 \sigma$ & Age & $1 \sigma$ & Age & $1 \sigma$ \\
\hline CDG01-01 & 300 & 577 & 0.52 & 0.255596 & 0.008384 & 0.034111 & 0.000382 & 231.1 & 6.8 & 216.2 & 2.4 \\
\hline CDG01-02 & 547 & 739 & 0.74 & 0.230797 & 0.006918 & 0.033233 & 0.000315 & 210.9 & 5.7 & 210.8 & 2.0 \\
\hline CDG01-03 & 400 & 744 & 0.54 & 0.230956 & 0.007554 & 0.033618 & 0.000356 & 211.0 & 6.2 & 213.2 & 2.2 \\
\hline CDG01-04 & 300 & 930 & 0.32 & 0.231304 & 0.006904 & 0.033856 & 0.000468 & 211.3 & 5.7 & 214.6 & 2.9 \\
\hline CDG01-05 & 211 & 460 & 0.46 & 0.247813 & 0.009077 & 0.034124 & 0.000352 & 224.8 & 7.4 & 216.3 & 2.2 \\
\hline CDG01-06 & 385 & 629 & 0.61 & 0.234334 & 0.007178 & 0.034473 & 0.000414 & 213.8 & 5.9 & 218.5 & 2.6 \\
\hline CDG01-07 & 226 & 445 & 0.51 & 0.243863 & 0.008634 & 0.033403 & 0.000311 & 221.6 & 7.0 & 211.8 & 1.9 \\
\hline CDG01-08 & 492 & 684 & 0.72 & 0.245532 & 0.007382 & 0.033634 & 0.000344 & 222.9 & 6.0 & 213.3 & 2.1 \\
\hline CDG01-09 & 1222 & 1069 & 1.14 & 0.219964 & 0.006578 & 0.031091 & 0.000292 & 201.9 & 5.5 & 197.4 & 1.8 \\
\hline CDG01-10 & 256 & 508 & 0.50 & 0.238907 & 0.008682 & 0.033818 & 0.0003 & 217.5 & 7.1 & 214.4 & 2.2 \\
\hline CDG01-11 & 416 & 714 & 0.58 & 0.246925 & 0.007703 & 0.033763 & 0.000 & 224.1 & 6.3 & 21 & 2.3 \\
\hline CDG01-12 & 176 & 428 & 0.41 & 0.250022 & 0.009 & 0.034307 & 0.000 & 226.6 & 7.5 & & 2.7 \\
\hline CDG01-13 & 194 & 484 & 0.40 & 0.232775 & 0.009352 & 0.033610 & 0.000 & 212 & 7.7 & & 2.5 \\
\hline CDG01-14 & 483 & 766 & 0.63 & 0.254202 & 0.008 & 0.0344 & 0.000 & 23 & 7.2 & & 2.7 \\
\hline CDG01-15 & 211 & 455 & & 0.243216 & 0.008780 & 0.03 & 0.000 & 22 & 7.2 & & 2.6 \\
\hline CDG01-16 & 131 & 349 & & 0.239577 & 0.0091 & & 0.00 & & & & 2.5 \\
\hline CDG01-17 & 271 & & & & & & & & & & 2.5 \\
\hline & & & & & & & & & & & 2.6 \\
\hline & 324 & & & & & & & & & & 2.5 \\
\hline CDG01-20 & 240 & & & & & & & & & & 2.4 \\
\hline CDG01-21 & 113 & & & & & & & & 7.5 & & 2.6 \\
\hline CDG01-22 & 229 & 470 & 0.49 & 0.238535 & 0.008811 & 0.033846 & 0.000 & 217.2 & 7.2 & 214.6 & 2.3 \\
\hline CDG01-23 & 151 & 388 & 0.39 & 0.226945 & 0.007936 & 0.033975 & 0.000 & 207.7 & 6.6 & 215.4 & 2.7 \\
\hline CDG & 229 & 470 & 0.49 & 0.221080 & 0.007490 & 0.033438 & 0.000 & 202.8 & 6.2 & 212.0 & 2.2 \\
\hline CDG01-25 & 180 & 357 & 0.50 & 0.244226 & 0.008381 & 0.034059 & 0.0003 & 221.9 & 6.8 & 215.9 & 2.4 \\
\hline CDGO & 253 & 442 & 0.57 & 0.234194 & 0.009242 & 0.033498 & 0.000375 & 213.7 & 7.6 & 212.4 & 2.3 \\
\hline CDG01-27 & 178 & 423 & 0.42 & 0.245466 & 0.009 & 0.033892 & 0.000 & 222.9 & 8.1 & 214.9 & 2.3 \\
\hline CDG01-28 & 281 & 576 & 0.49 & 0.241592 & 0.007813 & 0.034467 & 0.000325 & 219.7 & 6.4 & 218.4 & 2.0 \\
\hline CDG01-29 & 156 & 329 & 0.47 & 0.228488 & 0.009659 & 0.034469 & 0.000460 & 208.9 & 8.0 & 218.5 & 2.9 \\
\hline CDG01-30 & 192 & 465 & 0.41 & 0.236486 & 0.007728 & 0.033521 & 0.000318 & 215.5 & 6.3 & 212.5 & 2.0 \\
\hline GJ-1 & 9.43 & 307 & 0.03 & 0.7644 & 0.0255 & 0.0984 & 0.0011 & 577 & 14.65 & 605 & 6.59 \\
\hline GJ-1 & 9.37 & 308 & 0.03 & 0.7994 & 0.0251 & 0.0969 & 0.0011 & 596 & 14.19 & 596 & 6.30 \\
\hline GJ-1 & 9.64 & 297 & 0.03 & 0.8183 & 0.0213 & 0.0978 & 0.0010 & 607 & 11.91 & 602 & 5.97 \\
\hline GJ-1 & 9.86 & 298 & 0.03 & 0.8009 & 0.0223 & 0.0968 & 0.0010 & 597 & 12.59 & 596 & 5.75 \\
\hline GJ-1 & 9.41 & 297 & 0.03 & 0.7797 & 0.0234 & 0.0976 & 0.0011 & 585 & 13.35 & 600 & 6.75 \\
\hline GJ-1 & 9.57 & 302 & 0.03 & 0.7983 & 0.0241 & 0.0968 & 0.0011 & 596 & 13.59 & 596 & 6.32 \\
\hline GJ-1 & 9.66 & 298 & 0.03 & 0.8322 & 0.0217 & 0.0978 & 0.0010 & 615 & 12.02 & 601 & 5.95 \\
\hline GJ-1 & 9.58 & 299 & 0.03 & 0.7979 & 0.0240 & 0.0985 & 0.0010 & 596 & 13.58 & 606 & 5.66 \\
\hline
\end{tabular}



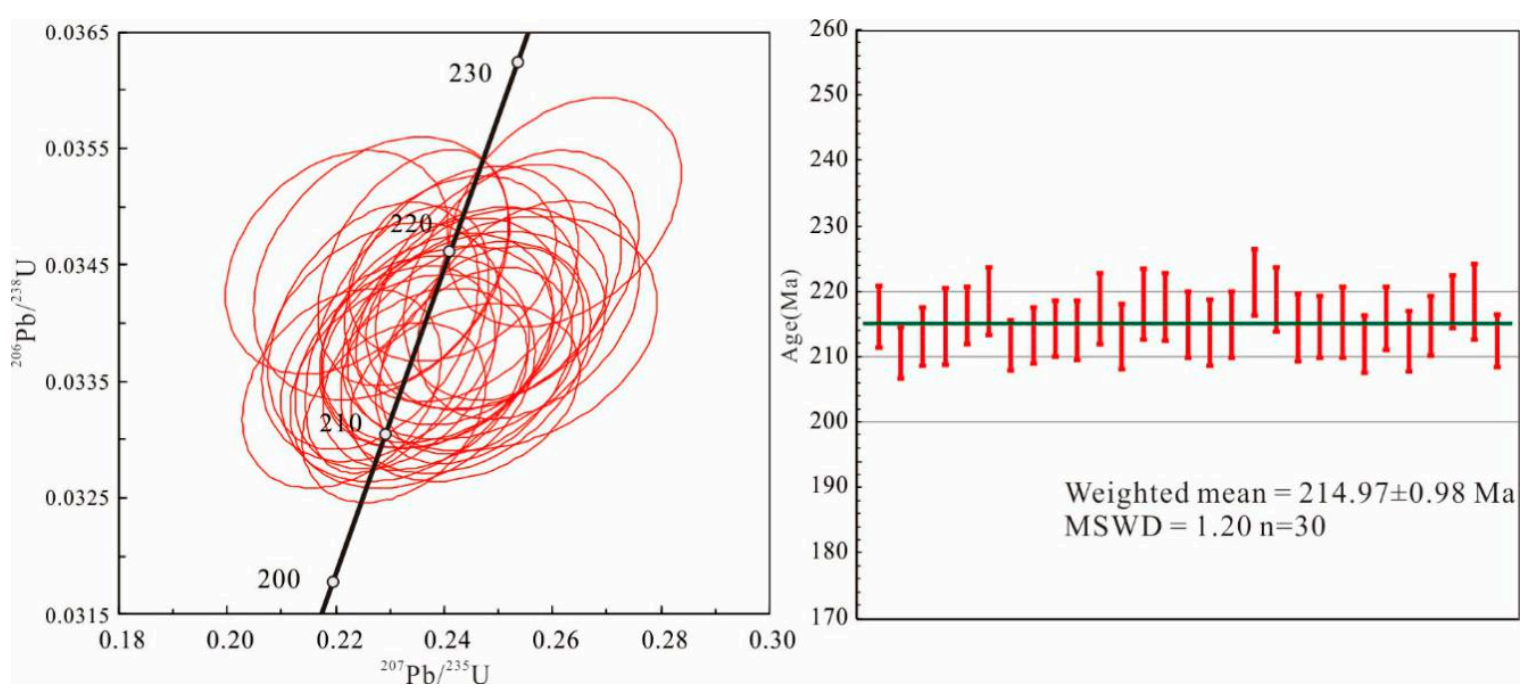

Figure 4. Zircon $\mathrm{U}-\mathrm{Pb}$ Concordia and ${ }^{206} \mathrm{~Pb} /{ }^{238} \mathrm{~Pb}$ weighted mean age diagrams for the Changdagou granodiorite porphyry.

\subsection{Zircon Trace Element Compositions}

Zircon trace elements data of the Changdagou granodiorite are presented in Table 2, and the chondrite-normalized REE (Rare Earth element) patterns are shown in Figure 5. All the zircon grains are LREE (Light Rare Earth Element)-depleted and HREE (Heavy Rare Earth Element)-enriched. Concentrations of $\mathrm{Ce}$ and $\mathrm{Eu}$ are of 3.4-106 ppm and 0.14-0.75 ppm, respectively. All zircon samples show strong positive Ce anomalies $\left(\mathrm{Ce}_{\mathrm{N}} / \mathrm{Ce}_{\mathrm{N}}{ }^{*}=14\right.$ to 172$)$ and slightly negative Eu anomalies $\left(\mathrm{Eu}_{\mathrm{N}}{ }^{*}=0.09\right.$ to 0.32$)$. These characters resemble those of typical magmatic zircon [48].

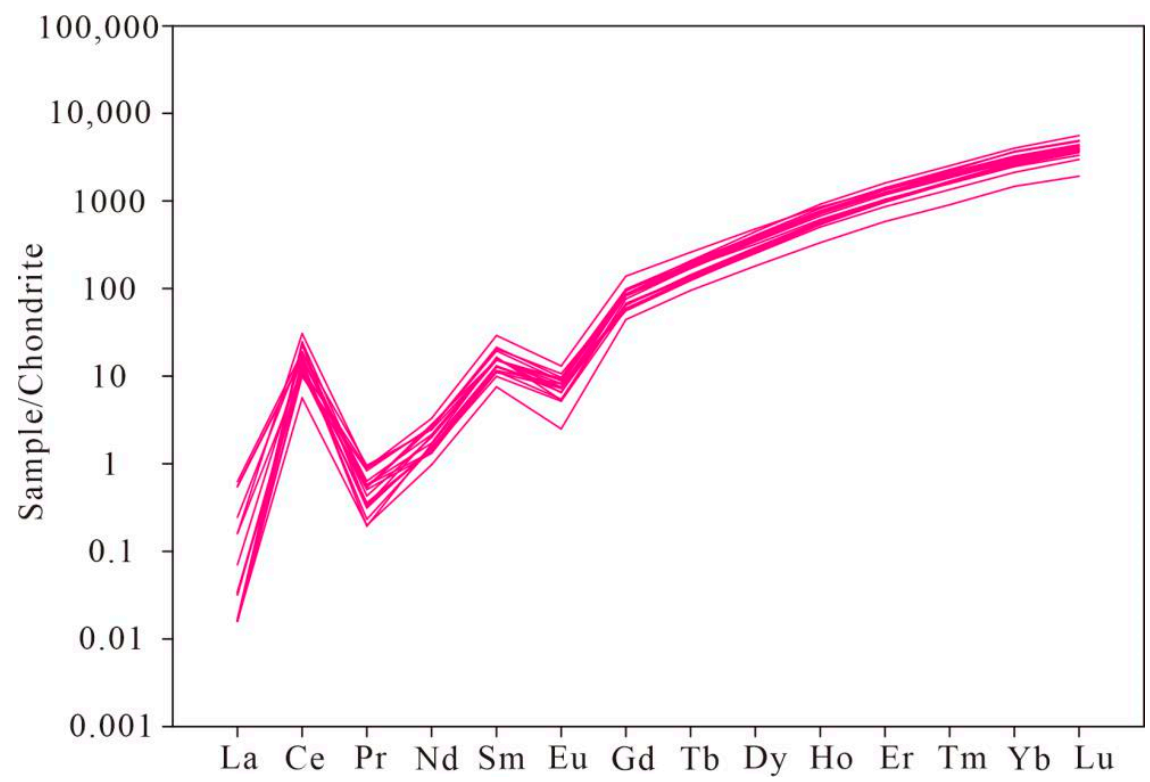

Figure 5. Chondrite-normalized REE diagram of zircon grains from the Changdagou granodiorite porphyry. Normalizing values from $[48,49]$. 
Table 2. Trace element concentrations of zircon grains from Changdagou granodiorite porphyry.

\begin{tabular}{|c|c|c|c|c|c|c|c|c|c|c|c|c|c|c|c|c|c|}
\hline Samples & Ti & $\mathbf{Y}$ & La & $\mathrm{Ce}$ & Pr & $\mathrm{Nd}$ & Sm & Eu & Gd & $\mathbf{T b}$ & Dy & Ho & Er & Tm & $\mathbf{Y b}$ & $\mathbf{P}$ & Hf \\
\hline CDG01-01 & 7.05 & 1324 & 0.132 & 9.89 & 0.06 & 1 & 2.41 & 0.38 & 17.58 & 6.93 & 99 & 44 & 221 & 54 & 555 & 382 & 10,978 \\
\hline CDG01-02 & 5.11 & 1211 & 10.41 & 33.41 & 2.78 & 11.86 & 4.07 & 0.74 & 19.02 & 6.74 & 90 & 38 & 195 & 47 & 493 & 605 & 11,436 \\
\hline CDG01-03 & 2.79 & 1587 & 0.004 & 15.15 & 0.05 & 1.21 & 2.98 & 0.51 & 19.26 & 7.74 & 113 & 52 & 265 & 64 & 681 & 398 & 12,002 \\
\hline CDG01-04 & 2.9 & 1256 & b.d. & 5.45 & 0.02 & 1.16 & 2.04 & 0.17 & 15.01 & 6.3 & 88 & 41 & 216 & 56 & 615 & 340 & 12,736 \\
\hline CDG01-05 & 4.21 & 1001 & 0.008 & 8.46 & 0.02 & 0.7 & 1.99 & 0.38 & 13.55 & 4.99 & 71 & 32 & 168 & 42 & 461 & 365 & 11,900 \\
\hline CDG01-06 & 3.64 & 1074 & 8.014 & 29.05 & 2.25 & 10.97 & 3.41 & 0.54 & 16.97 & 5.85 & 78 & 35 & 175 & 42 & 463 & 539 & 12,201 \\
\hline CDG01-07 & 2.23 & 1022 & 0.008 & 8.51 & 0.03 & 0.72 & 1.74 & 0.32 & 13.94 & 5.13 & 71 & 33 & 168 & 41 & 435 & 350 & 11,657 \\
\hline CDG01-08 & 2.84 & 1258 & 0.017 & 10.34 & 0.04 & 0.94 & 2.35 & 0.48 & 19.51 & 7.05 & 95 & 41 & 204 & 47 & 478 & 410 & 11,449 \\
\hline CDG01-09 & 4.16 & 1465 & 0.038 & 18.82 & 0.09 & 1.53 & 4.43 & 0.76 & 28.34 & 9.73 & 122 & 48 & 225 & 51 & 512 & 342 & 12,681 \\
\hline CDG01-10 & 2.05 & 873 & 0.008 & 6.67 & 0.03 & 0.66 & 1.52 & 0.3 & 11.96 & 4.69 & 64 & 29 & 143 & 34 & 359 & 589 & 12,359 \\
\hline CDG01-11 & 4.78 & 1092 & 44.713 & 106.16 & 12.5 & 55.33 & 12.01 & 0.51 & 24.76 & 7.2 & 88 & 36 & 175 & 41 & 416 & 341 & 11,535 \\
\hline CDG01-12 & 3.8 & 982 & 0.008 & 7.25 & 0.05 & 0.62 & 1.77 & 0.47 & 11.5 & 4.6 & 66 & 30 & 160 & 41 & 444 & 365 & 11,951 \\
\hline CDG01-13 & 3.98 & 1174 & b.d. & 7.7 & 0.02 & 0.83 & 1.96 & 0.3 & 13.97 & 5.73 & 83 & 37 & 194 & 48 & 508 & 390 & 12,297 \\
\hline CDG01-14 & 2.58 & 1292 & 0.004 & 14.02 & 0.03 & 0.98 & 2.33 & 0.52 & 17.23 & 6.77 & 95 & 41 & 208 & 51 & 538 & 363 & 11,644 \\
\hline CDG01-15 & 5597 & 1052 & 0.13 & 9.57 & 0.05 & 0.64 & 1.95 & 0.43 & 13.32 & 5.35 & 75 & 33 & 172 & 42 & 458 & 322 & 12,351 \\
\hline CDG01-16 & 3.68 & 721 & b.d. & 5.53 & 0.02 & 0.34 & 1.47 & 0.3 & 8.68 & 3.37 & 49 & 23 & 119 & 31 & 343 & 428 & 11,130 \\
\hline CDG01-17 & 4.03 & 1277 & 0.038 & 7.4 & 0.08 & 1.2 & 3.28 & 0.56 & 20.26 & 7.66 & 101 & 42 & 203 & 49 & 509 & 348 & 11,229 \\
\hline CDG01-18 & 3.18 & 750 & b.d. & 3.95 & 0.04 & 0.49 & 1.56 & 0.34 & 10.2 & 4.03 & 54 & 24 & 125 & 31 & 335 & 381 & 12,121 \\
\hline CDG01-19 & 4.1 & 1319 & 0.149 & 11.49 & 0.09 & 1.14 & 2.35 & 0.54 & 17.54 & 6.45 & 94 & 41 & 221 & 56 & 624 & 397 & 11,235 \\
\hline CDG01-21 & 2.74 & 593 & 0.004 & 3.48 & 0.02 & 0.46 & 1.16 & 0.14 & 9.07 & 3.55 & 46 & 19 & 96 & 23 & 249 & 622 & 11,447 \\
\hline CDG01-22 & 2.97 & 1206 & 1.921 & 13.54 & 0.63 & 3.35 & 3.1 & 0.52 & 16.54 & 5.87 & 83 & 38 & 194 & 48 & 520 & 304 & 12,036 \\
\hline CDG01-23 & 2.23 & 794 & b.d. & 5.98 & 0.03 & 0.61 & 1.35 & 0.25 & 10.64 & 4.02 & 54 & 25 & 129 & 33 & 358 & 378 & 11,304 \\
\hline CDG01-24 & 1.52 & 1272 & 0.004 & 9.26 & 0.02 & 0.78 & 2.01 & 0.42 & 16.87 & 6.37 & 89 & 40 & 206 & 50 & 523 & 338 & 11,157 \\
\hline CDG01-25 & 2.36 & 1081 & 0.004 & 6.08 & 0.08 & 1.23 & 3.12 & 0.62 & 17.13 & 6.6 & 81 & 35 & 172 & 40 & 418 & 415 & 10,100 \\
\hline CDG01-26 & 4.95 & 1635 & b.d. & 9.11 & 0.05 & 1.18 & 2.46 & 0.58 & 19.9 & 8.29 & 120 & 54 & 263 & 62 & 643 & 335 & 11,568 \\
\hline CDG01-27 & 3.43 & 1014 & 0.008 & 8.15 & 0.03 & 0.67 & 1.69 & 0.42 & 12.22 & 4.86 & 68 & 32 & 167 & 43 & 468 & 387 & 11,428 \\
\hline CDG01-28 & 2.91 & 1444 & 0.058 & 11.59 & 0.05 & 1.3 & 2.38 & 0.44 & 17.82 & 7.31 & 103 & 47 & 234 & 58 & 614 & 305 & 11,514 \\
\hline CDG01-29 & 1.93 & 687 & b.d. & 4.78 & 0.03 & 0.48 & 1.25 & 0.2 & 8.83 & 3.6 & 49 & 22 & 113 & 27 & 297 & 349 & 11,722 \\
\hline CDG01-30 & 3.62 & 1184 & 0.004 & 6.47 & 0.05 & 0.8 & 2.52 & 0.31 & 15.59 & 6.39 & 87 & 38 & 194 & 47 & 492 & 437 & 11,958 \\
\hline
\end{tabular}

Note: "b.d." represent the concentration below the detection limit. 
The calculated $\log f \mathrm{O}_{2}$ values are listed in Table A1 and plotted in Figure 6a. In the $\log f \mathrm{O}_{2}-\mathrm{T}$ diagram, the Changdagou granodiorite porphyry data plot between the FMQ and MH buffers. For the data from the Pulang and Lannitang PCDs (southern Yidun arc), the Lannitang porphyry samples mainly plot above the NNO buffer, whilst half of the Pualng samples plot above the MH buffer. Therefore, the Pulang (FMQ -9.25 to +13.22) and Lannitang (FMQ +0.23 to +9.52) granites have higher $\log f \mathrm{O}_{2}$ than the Changdagou granites (FMQ -3.14 to +7.44 ) (Figure 6b). Zircon grains of Changdgou granodiorite have relatively low Ti contents (1.51-7.04 ppm), which gave Ti-in-zircon temperatures of 628 to $757^{\circ} \mathrm{C}$ (avg. $689^{\circ} \mathrm{C}$ ). In the $\log f \mathrm{O}_{2}-\mathrm{T}$ diagram, the Changdgou granodiorite has a lower Ti-in-zircon temperature than those of the Pulang and Lannitang granites.
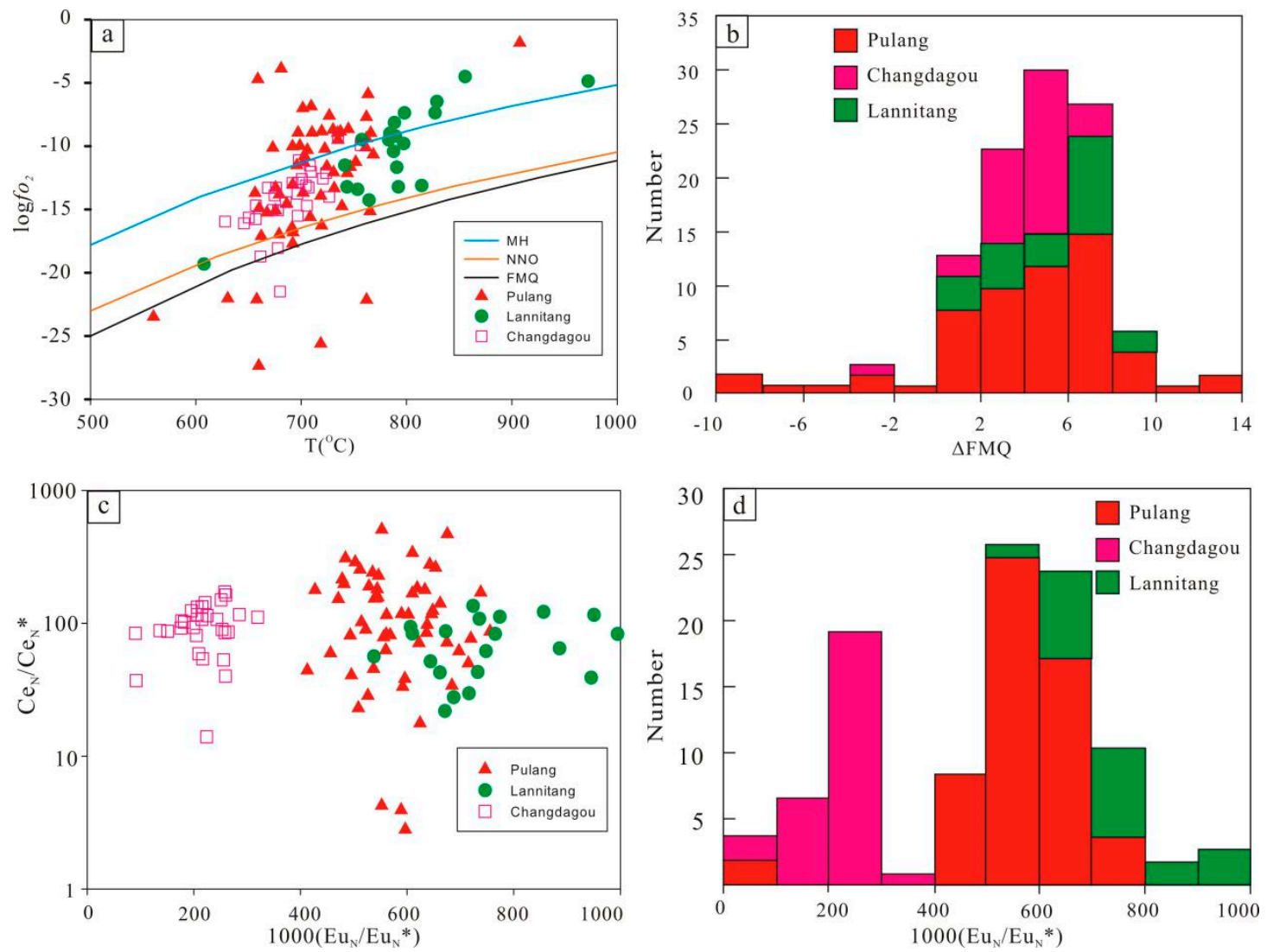

Figure 6. $\mathrm{Ce}_{\mathrm{N}} / \mathrm{Ce}_{\mathrm{N}}{ }^{*}$-related binary diagrams of zircon grains for the Changdagou, Pulang and Lannitang granites from Yidun arc. (a) $\log f \mathrm{O}_{2}$ vs. temp. ${ }^{\circ} \mathrm{C}$ diagram, where $\log f \mathrm{O}_{2}$ value was calculated by the method of Trail et al. [34]; (b) Histogram of oxygen fugacity; (c) $\mathrm{Ce}_{\mathrm{N}} / \mathrm{Ce}_{\mathrm{N}}{ }^{*} \mathrm{vs}$. $\mathrm{Eu}_{\mathrm{N}} / \mathrm{Eu}_{\mathrm{N}}{ }^{*}$ plot, where $\mathrm{Ce}_{\mathrm{N}}{ }^{*}$ is calculated by fitting method; $(\mathbf{d})$ Histogram of $1000\left(\mathrm{Eu}_{\mathrm{N}} / \mathrm{Eu}_{\mathrm{N}}{ }^{*}\right)$ vs. Data are as listed in Table A1.

As shown in Figure $6 c, d$, Changdagou granite shows a lower and narrower range of zircon $\mathrm{Eu}_{\mathrm{N}} / \mathrm{Eu}_{\mathrm{N}}{ }^{*}$ values $(0.1-0.32$; avg. 0.22) than those of the Lannitang (0.54-0.99; avg. 0.75) and Pulang (0.41-0.76; avg. 0.56) granites. Zircon $\mathrm{Ce}_{\mathrm{N}} / \mathrm{Ce}_{\mathrm{N}}{ }^{*}$ values of the Changdagou sample (14-172; avg. 98) have a similar range with their Lannitang (22-135; avg. 73) and Pulang (3-507; avg. 143) counterparts (Table A1). Besides, it was found that the $\mathrm{Ce}_{\mathrm{N}} / \mathrm{Ce}_{\mathrm{N}}{ }^{*}$ and $\mathrm{Eu}_{\mathrm{N}} / \mathrm{Eu}_{\mathrm{N}}{ }^{*}$ does not have any positive relationship.

The zircon $\mathrm{Eu}_{\mathrm{N}} / \mathrm{Eu}_{\mathrm{N}}{ }^{*}$ vs. $(\mathrm{Ce} / \mathrm{Nd}) / \mathrm{Y}$ diagram shows marked distinction between the Changdagou and Pulang granites (Lannitang granites not shown due to the lack of published zircon $Y$ data). The Changdagou granites show lower zircon $(\mathrm{Ce} / \mathrm{Nd}) / \mathrm{Y}$ ratios $(0.002-0.022 ;$ avg. 0.009$)$ than their Pulang counterparts (0.007-0.18; avg. 0.038), and there is a positive correlation between $\mathrm{Eu}_{\mathrm{N}} / \mathrm{Eu}_{\mathrm{N}}{ }^{*}$ and $(\mathrm{Ce} / \mathrm{Nd}) / \mathrm{Y}$ ratios (Figure 7$)$. 

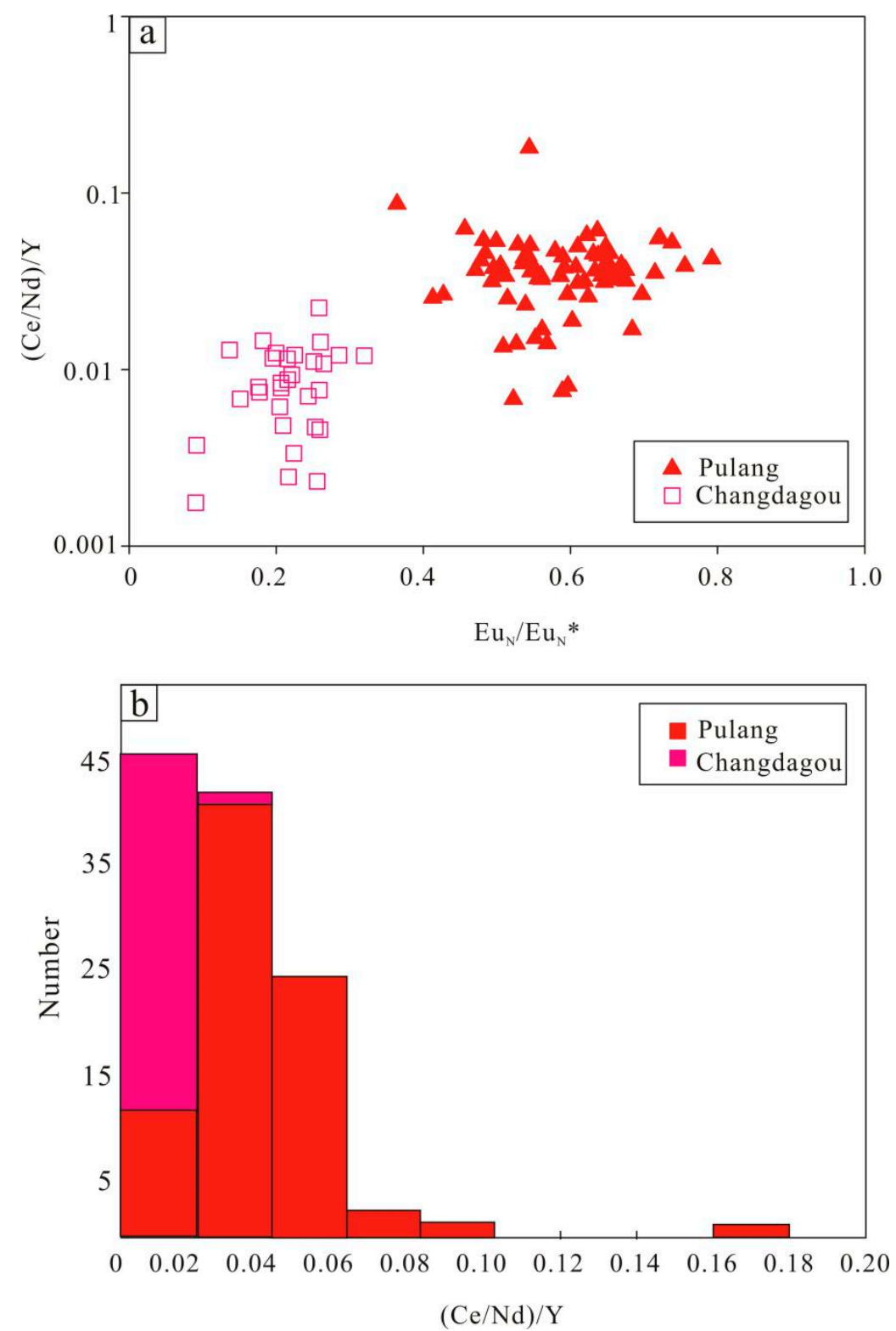

Figure 7. (a) $(\mathrm{Ce} / \mathrm{Nd}) / \mathrm{Y}$ vs. $\mathrm{Eu}_{\mathrm{N}} / \mathrm{Eu}_{\mathrm{N}}{ }^{*}$ diagram of zircon grains for the Changdagou and Pulang porphyries; (b) histogram of $(\mathrm{Ce} / \mathrm{Nd}) / \mathrm{Y}$ values.

\subsection{Zircon Hf Isotopes}

Hf-isotopic data of the zircon grains analyzed from the Changdagou granodiorite porphyry (CDG01) are shown in Table 3. Zircon GJ-1 and 91,500 was used as the reference standard, with weighted mean ${ }^{176} \mathrm{Hf} /{ }^{177} \mathrm{Hf}$ ratios of $0.282017 \pm 0.0000060$ and $0.282308 \pm 0.0000035$, respectively, determined by our routine analyses. The zircon $\varepsilon \mathrm{Hf}(\mathrm{t})$ values vary in a narrow range $(-2.9$ to -4.9 ; avg. -3.4), corresponding to the lower crust (Figure 8a). Comparatively, the Pulang granites have higher zircon $\varepsilon \mathrm{Hf}(\mathrm{t})$ values (-4.7 to 2.5 ; [6]), which plot above the Chondrite Uniform Reservoir (CHUR) evolutionary line in the $\varepsilon \mathrm{Hf}(\mathrm{t}) \mathrm{vs}$. U-Pb age diagram (Figure 8). 
Table 3. Hf isotopes in zircon grains from Changdagou granodiorite porphyry.

\begin{tabular}{|c|c|c|c|c|c|c|c|c|c|c|}
\hline Samples & ${ }^{176} \mathrm{Hf} /{ }^{177} \mathrm{Hf}$ & $1 \sigma$ & ${ }^{176} \mathrm{Lu} /{ }^{177} \mathrm{Hf}$ & $1 \sigma$ & ${ }^{176} \mathrm{Yb} /{ }^{177} \mathrm{Hf}$ & $1 \sigma$ & $\varepsilon \mathbf{H f}(\mathbf{0})$ & $\varepsilon H f(t)$ & TDM(Ma) & TDMC(Ma) \\
\hline CDG01-01 & 0.282549 & 0.000008453 & 0.001543 & 0.00002525 & 0.041931 & 0.000585 & -8.33 & -3.78 & 1009 & 1466 \\
\hline CDG01-02 & 0.282553 & 0.000007740 & 0.001566 & 0.00001218 & 0.041916 & 0.000406 & -8.22 & -3.66 & 1005 & 1458 \\
\hline CDG01-03 & 0.282568 & 0.000007793 & 0.001337 & 0.00001767 & 0.036474 & 0.000564 & -7.66 & -3.08 & 976 & 1421 \\
\hline CDG01-05 & 0.282573 & 0.000008512 & 0.001580 & 0.00000861 & 0.042021 & 0.000185 & -7.48 & -2.93 & 975 & 1412 \\
\hline CDG01-06 & 0.282556 & 0.000008577 & 0.001426 & 0.00000996 & 0.039120 & 0.000397 & -8.09 & -3.51 & 996 & 1449 \\
\hline CDG01-07 & 0.282569 & 0.000008165 & 0.001459 & 0.00000939 & 0.039605 & 0.000191 & -7.63 & -3.06 & 978 & 1420 \\
\hline CDG01-08 & 0.282556 & 0.000007987 & 0.001472 & 0.00001568 & 0.039988 & 0.000302 & -8.09 & -3.52 & 997 & 1450 \\
\hline CDG01-11 & 0.282520 & 0.000008477 & 0.001970 & 0.00004212 & 0.057167 & 0.001163 & -9.37 & -4.88 & 1063 & 1536 \\
\hline CDG01-12 & 0.282565 & 0.000007780 & 0.001549 & 0.00003119 & 0.042051 & 0.000783 & -7.77 & -3.21 & 986 & 1430 \\
\hline CDG01-13 & 0.282561 & 0.000009007 & 0.001495 & 0.00001240 & 0.039560 & 0.000151 & -7.93 & -3.36 & 991 & 1439 \\
\hline CDG01-15 & 0.282574 & 0.000008591 & 0.001298 & 0.00001065 & 0.035959 & 0.000402 & -7.47 & -2.87 & 967 & 1408 \\
\hline CDG01-16 & 0.282575 & 0.000007749 & 0.000731 & 0.00001281 & 0.019467 & 0.000398 & -7.41 & -2.74 & 951 & 1400 \\
\hline CDG01-19 & 0.282561 & 0.000008847 & 0.001569 & 0.00001555 & 0.043378 & 0.000470 & -7.93 & -3.37 & 993 & 1440 \\
\hline CDG01-20 & 0.282560 & 0.000008137 & 0.001528 & 0.00002673 & 0.042351 & 0.000834 & -7.94 & -3.39 & 993 & 1441 \\
\hline CDG01-21 & 0.282570 & 0.000007521 & 0.000859 & 0.00000944 & 0.023062 & 0.000150 & -7.62 & -2.96 & 962 & 1414 \\
\hline CDG01-22 & 0.282546 & 0.000007604 & 0.001355 & 0.00000275 & 0.037267 & 0.000181 & -8.44 & -3.86 & 1008 & 1471 \\
\hline CDG01-25 & 0.282560 & 0.000007855 & 0.001161 & 0.00000818 & 0.031958 & 0.000242 & -7.97 & -3.36 & 984 & 1439 \\
\hline CDG01-26 & 0.282538 & 0.000009456 & 0.002075 & 0.00003579 & 0.057039 & 0.000774 & -8.73 & -4.24 & 1039 & 1496 \\
\hline CDG01-27 & 0.282549 & 0.000008600 & 0.001454 & 0.00002130 & 0.039899 & 0.000614 & -8.33 & -3.76 & 1006 & 1465 \\
\hline CDG01-30 & 0.282565 & 0.000007378 & 0.001037 & 0.00001720 & 0.028808 & 0.000593 & -7.78 & -3.15 & 973 & 1426 \\
\hline
\end{tabular}

Note: $\left.\left.\varepsilon \mathrm{Hf}(\mathrm{t})=10,000 \times\left\{\left[{ }^{176} \mathrm{Hf} /{ }^{177} \mathrm{Hf}\right)_{\mathrm{S}}-\left({ }^{176} \mathrm{Lu} /{ }^{177} \mathrm{Hf}\right)_{\mathrm{S}} \times\left(\mathrm{e}^{\lambda t}-1\right)\right] /\left({ }^{176} \mathrm{Hf} /{ }^{177} \mathrm{Hf}\right) \mathrm{CHUR} .0-\left({ }^{176} \mathrm{Lu} /{ }^{177} \mathrm{Hf}\right) \mathrm{CHUR} \times\left(\mathrm{e}^{\lambda t}-1\right)\right]-1\right\}$. TDM $=1 / \lambda \times \ln \left\{1+\left[\left({ }^{176} \mathrm{Hf} /{ }^{177} \mathrm{Hf}\right)_{\mathrm{S}}-\left({ }^{176} \mathrm{Hf} /{ }^{177} \mathrm{Hf}\right)_{\mathrm{DM}}\right] /\right.$ $\left.\left.\left[\left({ }^{176} \mathrm{Lu} /{ }^{177} \mathrm{Hf}\right)_{S}-{ }^{176} \mathrm{Lu} /{ }^{177} \mathrm{Hf}\right) \mathrm{DM}\right]\right\} . \mathrm{TDM}_{2}=t_{\mathrm{DM}}-\left(t_{\mathrm{DM}}-\mathrm{t}\right) \times\left[\left(f \mathrm{fc}-f_{\mathrm{s}}\right) /\left(f_{c c}-f_{\mathrm{DM}}\right)\right] .\left({ }^{176} \mathrm{Lu} /{ }^{177} \mathrm{Hf}\right)_{\mathrm{CHUR}}=0.0332,\left({ }^{176} \mathrm{Hf} /{ }^{177} \mathrm{Hf}\right)_{\mathrm{CHUR}}=0.282772,\left({ }^{176} \mathrm{Lu} /{ }^{177} \mathrm{Hf}\right){ }_{\mathrm{DM}}=0.0384 \mathrm{and}\left({ }^{176} \mathrm{Hf} /{ }^{177} \mathrm{Hf}\right)$ $\mathrm{DM}=0.28325 ;\left({ }^{176} \mathrm{Lu} /{ }^{177} \mathrm{Hf}\right)$ mean crust $=0.015 . f c c=\left[\left({ }^{176} \mathrm{Lu} /{ }^{177} \mathrm{Hf}\right)\right.$ mean crust $\left.\left./\left({ }^{176} \mathrm{Lu} /{ }^{177} \mathrm{Hf}\right)_{\mathrm{CHUR}}\right]-1 ; f_{s}=\mathrm{f}_{\mathrm{Lu}} / \mathrm{Hf} ; \mathrm{f}_{\mathrm{DM}}=\left[\left({ }^{176} \mathrm{Lu} /{ }^{177} \mathrm{Hf}\right)_{\mathrm{DM}} /{ }^{176} \mathrm{Lu} /{ }^{177} \mathrm{Hf}\right) \mathrm{CHUR}\right]-1 ; \mathrm{t}=\mathrm{crystallization} \mathrm{time}$ of zircon [51,52]. 

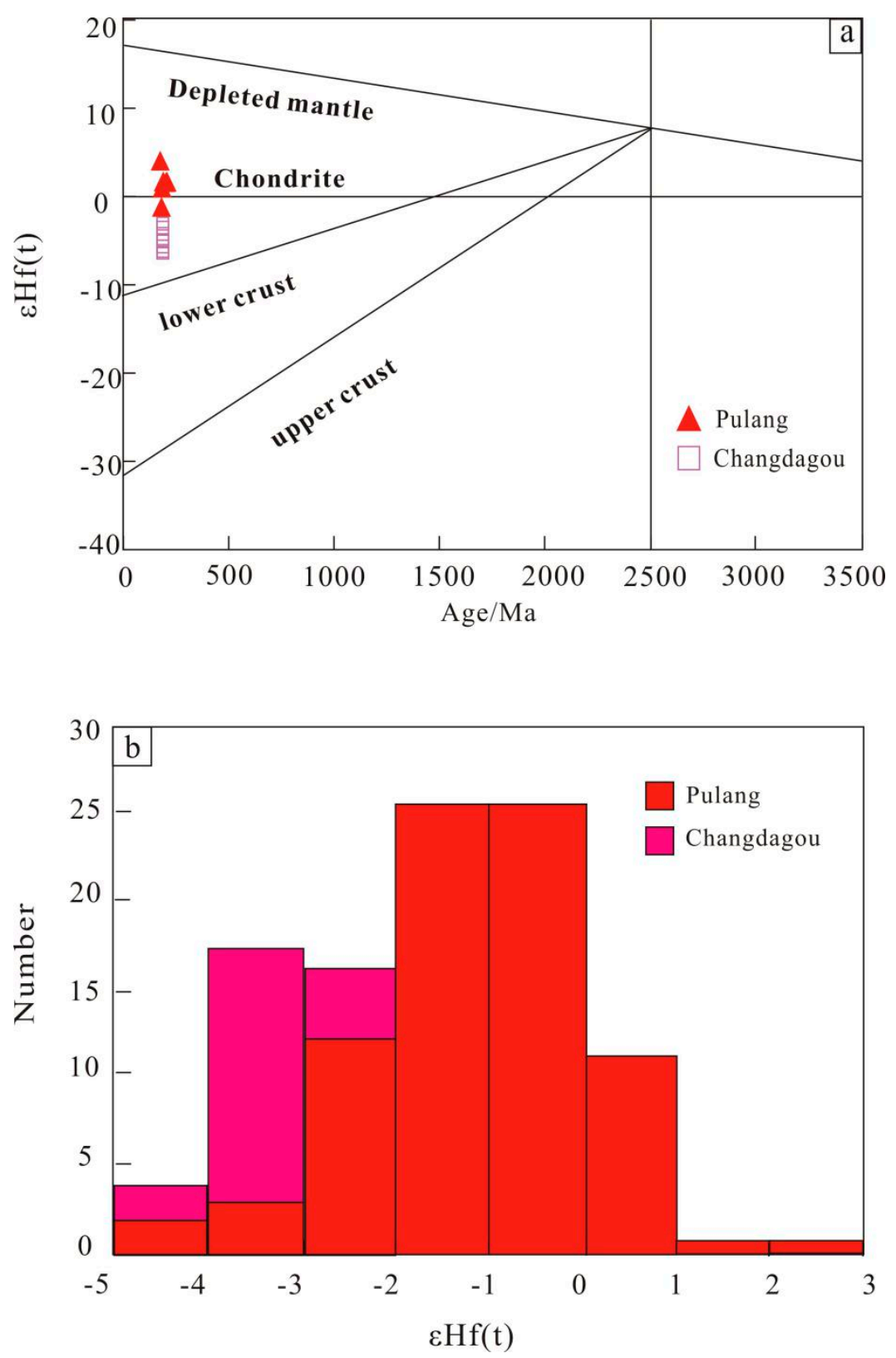

Figure 8. (a) Diagram of $\varepsilon H f(t)$ vs. U-Pb ages. (b) Histogram of $\varepsilon H f(t)$ values.

\section{Discussion}

\subsection{Geochronological Data}

The age of Changdagou granite has long been estimated by its intrusive relationship with the regional stratigraphy [53]. In this study, we report the first zircon $\mathrm{U}-\mathrm{Pb}$ age for the ore-forming Changdagou granodiorite porphyry $(214.97 \pm 0.98 \mathrm{Ma})$ from the northern Yidun arc. In the southern Yidun arc, Wang et al. [6] reported three zircon U-Pb ages $(214.3 \pm 2.9 \mathrm{Ma}, 211.6 \pm 3.1 \mathrm{Ma}$, and $214.1 \pm 2.9 \mathrm{Ma}$ ) for the Pulang complex, Chen et al. [54] reported one zircon U-Pb age (216.7 $\pm 1.2 \mathrm{Ma})$ for the Lannitang porphyry, and Ren et al. [55] reported one zircon U-Pb age (213.4 $\pm 1.5 \mathrm{Ma})$ for the Xuejiping porphyry. All these ages, no matter whether from the northern or southern segment of the Yidun arc, fall consistently into the Late Triassic, coeval with the Indosinian Orogeny in SW China and mainland SE Asia. With the lithological and geochemical features of the igneous-sedimentary assemblages in the region, it is broadly accepted that the Yidun zone was a continental arc developed 
on the Zhongza Block [2,4]. Thus, formation of the Changdagou granodiorite porphyry was probably related to the subduction associated with Late Triassic magmatism in the Yidun zone $[23,56,57]$.

\subsection{Magma Redox State}

It is generally accepted that oxidized magmas are favorable to the formation of PCDs, via inhibiting early sulfide precipitation and allowing the ore metals to be concentrated into the residual melt and hydrothermal fluids [11,58-60]. Shen et al. [58] argued that the oxygen fugacity has a positive relationship with the size of $\mathrm{PCDs}$ in the $\mathrm{CAOB}$, and that the $(\mathrm{NNO}+2)$ values can distinguish large and intermediate PCDs from small ones. In this study, the average magmatic oxygen fugacity (FMQ +3.98) and $\mathrm{Ce}_{\mathrm{N}} / \mathrm{Ce}_{\mathrm{N}}{ }^{*}(98)$ for the Changdagou granodiorite are much higher than the lowest limit (FMQ + 1.5) for porphyry $\mathrm{Cu}-(\mathrm{Au})-\mathrm{Mo}$ mineralization [61-63]. These data suggest that Changdagou granodiorite has PCD-forming potential. Meanwhile, the Pulang and Lannitang granites both contain higher $\log f \mathrm{O}_{2}$ values than those of the Changdagou granodiorite (Figure 6a,b), which may explain why the PCDs at Pulang (1.14 Mt) and Lannitang (0.30 Mt for Xuejiping) are larger than that at Changdagou [7].

\subsection{Magma Water Content}

High magmatic water contents are widely accepted to be important in enhancing the magma PCD fertility [38,39,41,59-63]. Lu et al. [14] suggested that the fertile magmas commonly contain high $(\mathrm{Ce} / \mathrm{Nd}) / \mathrm{Y}$ and high $\mathrm{Eu}_{\mathrm{N}} / \mathrm{Eu}_{\mathrm{N}}{ }^{*}$ ratios. However, $\mathrm{Eu}_{\mathrm{N}} / \mathrm{Eu}_{\mathrm{N}}{ }^{*}$ of zircon grain is also dependent on the magma oxidation state $[5,31,40,46]$. In this study, the lack of positive $C e_{N} / C_{N}{ }^{*} v s$. $E u_{N} / E_{N}{ }^{*}$ correlation, and the presence of positive $\mathrm{Eu}_{\mathrm{N}} / \mathrm{Eu}_{\mathrm{N}}{ }^{*}$ vs. $(\mathrm{Ce} / \mathrm{Nd}) / \mathrm{Y}$ correlation suggest that $\mathrm{Eu}_{\mathrm{N}} / \mathrm{Eu}_{\mathrm{N}}{ }^{*}$ is probably affected by water content (Figures $6 \mathrm{c}$ and $7 \mathrm{a}$ ). The Changdagou granodiorite porphyry has a narrower and lower $\mathrm{Eu}_{\mathrm{N}} / \mathrm{Eu}_{\mathrm{N}}{ }^{*}$ zircon grain range than those of the Lannitang and Pulang granites, which suggest that the latter two have higher magmatic water contents.

\subsection{Tectono-Metallogenic Implications on the Northern and Southern Yidun Arc}

It has been generally believed that high oxidization of arc magmas are related to subduction: the closer the distance of the arc magmas from the subduction zone, the higher the oxygen fugacity $[10,12,37,43,64-66]$. This is likely caused by the larger amount of oxidized ions (i.e., $\mathrm{Fe}^{3+}$, $\mathrm{Mn}^{4+}, \mathrm{S}^{6+}$, and $\mathrm{C}^{4+}$ ) carried by the slab-derived fluids closer to the subduction front [10,67-70]. In this study, we suggest that ore-forming magmas at Lannitang and Pulang (southern Yidun arc) have had higher water content and oxygen fugacity than those at Changdaou (northern Yidun arc), which implies that the southern Yidun arc was closer to the Ganzi-Litang subduction zone. A similar conclusion was also reached by Wang et al. [6].

In contrast with $\mathrm{Lu}, \mathrm{Hf}$ partitions into silicate melts more efficiently, melts derived from the magma would become less radiogenic (lower ${ }^{176} \mathrm{Lu} /{ }^{176} \mathrm{Hf}$ ) and thus have lower ${ }^{176} \mathrm{Hf} /{ }^{177} \mathrm{Hf}$ ratios relative to the residue [54,71]. Regional intrusions sourced from the continental crust generally possess low $\varepsilon \operatorname{Hf}(t)$ values, such as Hongshan quartz monzonite [6]. The Changdagou sample plot is close to the chondrite line and presents variations in $\varepsilon \mathrm{Hf}(t)$ values (Figure $8 \mathrm{a}, \mathrm{b}$ ). It is arranged with the coeval Late Triassic intrusions in the Songpan-Ganzi terrane [72].

As shown in Figure 9, most of the Late Triassic intrusions in the southern Yidun arc, including the Xuejiping, Pulang, Lannitang, and Songnuo intrusions, are PCD-forming, but coeval granites in the northern Yidun arc are mostly ore-barren, such as the Daocheng and Cuojiaoma intrusions [3]. These infertile rocks show a wide range of negative $\varepsilon \mathrm{Hf}(t)$ values, indicating a mixed source dominated by crustal components $[73,74]$. Unlike most other Late Triassic intrusions in the northern Yidun arc, the $\varepsilon \operatorname{Hf}(t)$ values of Changdagou granodiorite porphyry presents a narrow range which cluster around 0 , indicating a more mantle-derived source. The decreasing $\varepsilon \mathrm{Hf}(t)$ trend from south to north (and reverses sharply around Changdagou) in the Yidun arc probably indicate decreasing mantle-derived input to the granitoid formation from south to north, which may be attributed to the shape of the continental arc and/or subduction angle variation $[3,4,6]$. 


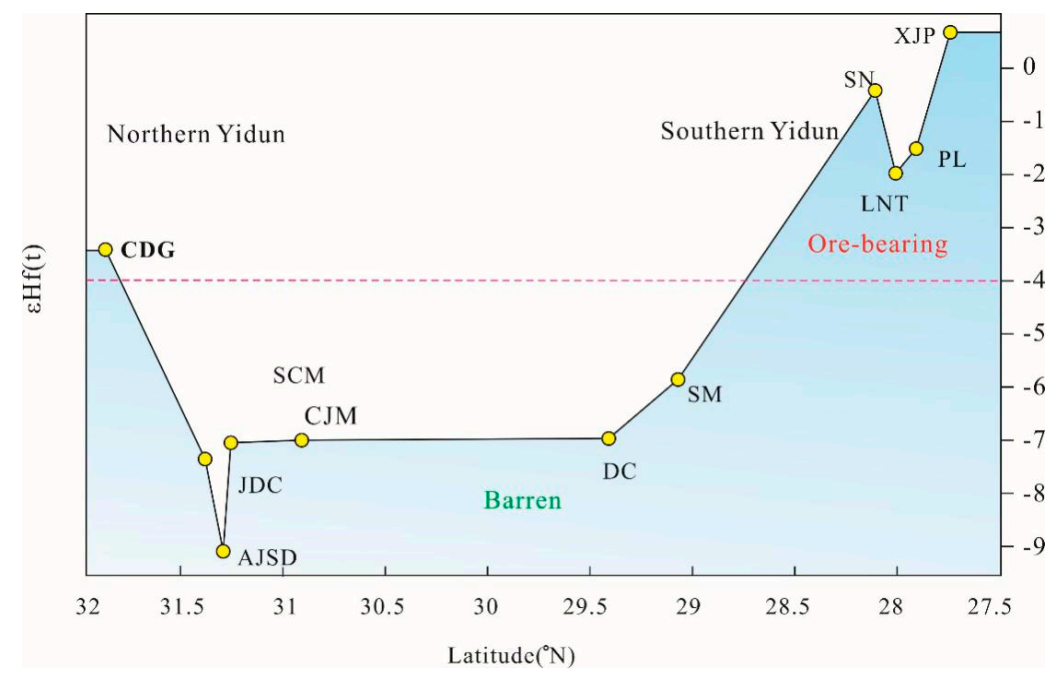

Figure 9. Diagram of $\varepsilon \mathrm{Hf}(t)$ vs. latitude to illustrate the contribution of mantle components for the ore-fertile granites from north to south in the Yidun arc. The data are from refs. [4,6]. Abbreviations: CDG-Changdagou; SCM-Sucuoma; AJSD-Ajisenduo; JDC-Jiaduocuo; CJM-Cuojiama; LNT—Lannitang; PL—Pulang; SM—Shenmu; SN—Songnuo; XJP—Xuejiping; DC—Daocheng.

\section{Conclusions}

1. Zircon $\mathrm{U}-\mathrm{Pb}$ dating of the Changdagou granodiorite porphyry in the northern Yidun arc yielded $214.97 \pm 0.98 \mathrm{Ma}$. This age is very similar to other Late Triassic PCD-forming magmatisms in the Yidun arc.

2. As reflected by proxies such as $\log f \mathrm{O}_{2}, \mathrm{Eu}_{\mathrm{N}} / \mathrm{Eu}_{\mathrm{N}}{ }^{*}$, and $(\mathrm{Ce} / \mathrm{Nd}) / \mathrm{Y}$, magmas that formed the Changdagou granodiorite porphyry may have had high oxygen fugacity (FMQ +3.98$)$ and water content, yet they were likely lower than those of the Pulang and Langnitang ore-causative porphyries. This may have limited the PCD size at Changdagou.

3. The southern segment of the Yidun arc was probably closer to the subduction front than the northern segment, where Changdagou is located. The more subduction-distal setting of the northern Yidun arc may have resulted in the lower oxygen fugacity, water content, enriched-mantle input, and thus the PCD-fertility there.

Author Contributions: X.-Y.L. and H.S. conceived and designed the experiments; X.-Y.L. performed the experiments; J.-R.Z. and X.-Y.L. analyzed the data; X.-Y.L. wrote the paper, assisted by all other authors; and C.-K.L. revised the paper.

Funding: This research was funded by the NSFC-China (418030401 and 41802251), the Geological Survey of China (1212011220391), and the China Scholarship Council Fund (201406380063).

Acknowledgments: We are grateful to Wei Gao for helping with the LA-ICP-MS analyses. Also, we appreciate the constructive comments and suggestions by the editor and three anonymous reviewers, with which the paper was greatly improved.

Conflicts of Interest: The authors declare no conflict of interest. 


\section{Appendix A}

Table A1. Crystallization temperatures, Eu and Ce anomalies, and magma oxygen fugacity of zircon grains from Changdagou, Pulang, and Lannitang granites.

\begin{tabular}{|c|c|c|c|c|c|c|}
\hline Spot. No & $\mathrm{T}$ (Ti in Zircon) & $\mathrm{CeN} / \mathrm{CeN}^{*}$ & $\log f \mathrm{O}_{2}$ & $\delta F M Q$ & $\mathrm{EuN} / \mathrm{EuN}^{*}$ & $\mathrm{Ce} / \mathrm{Nd} / \mathrm{Y}$ \\
\hline \multicolumn{7}{|c|}{ Changdagou Granite } \\
\hline CDG01-01 & 757 & 104 & -9.92 & 6.29 & 0.18 & 0.00744 \\
\hline CDG01-02 & 727 & 53 & -14.01 & 3 & 0.26 & 0.00233 \\
\hline CDG01-03 & 675 & 116 & -13.88 & 4.62 & 0.21 & 0.00788 \\
\hline CDG01-04 & 678 & 37 & -18.06 & 0.35 & 0.09 & 0.00373 \\
\hline CDG01-05 & 709 & 114 & -12.03 & 5.46 & 0.23 & 0.01207 \\
\hline CDG01-06 & 697 & 54 & -15.5 & 2.34 & 0.22 & 0.00247 \\
\hline CDG01-07 & 657 & 124 & -14.68 & 4.37 & 0.2 & 0.01158 \\
\hline CDG01-08 & 676 & 133 & -13.28 & 5.17 & 0.22 & 0.00877 \\
\hline CDG01-09 & 708 & 132 & -11.53 & 5.98 & 0.21 & 0.00838 \\
\hline CDG01-10 & 650 & 107 & -15.64 & 3.62 & 0.22 & 0.01149 \\
\hline CDG01-11 & 721 & 84 & -12.56 & 4.61 & 0.09 & 0.00176 \\
\hline CDG01-12 & 701 & 111 & -12.6 & 5.14 & 0.32 & 0.01198 \\
\hline CDG01-13 & 705 & 92 & -13.1 & 4.52 & 0.18 & 0.00795 \\
\hline CDG01-14 & 668 & 150 & -13.29 & 5.41 & 0.25 & 0.01111 \\
\hline CDG01-15 & 735 & 163 & -9.34 & 7.44 & 0.26 & 0.01429 \\
\hline CDG01-16 & 698 & 172 & -11.1 & 6.72 & 0.26 & 0.02233 \\
\hline CDG01-17 & 705 & 59 & -14.72 & 2.88 & 0.21 & 0.00482 \\
\hline CDG01-18 & 685 & 86 & -14.42 & 3.76 & 0.26 & 0.01078 \\
\hline CDG01-19 & 707 & 85 & -13.27 & 4.29 & 0.26 & 0.00766 \\
\hline CDG01-20 & 698 & 107 & -12.88 & 4.93 & 0.24 & 0.00706 \\
\hline CDG01-21 & 673 & 88 & -15.04 & 3.51 & 0.14 & 0.01289 \\
\hline CDG01-22 & 680 & 14 & -21.48 & -3.14 & 0.22 & 0.00335 \\
\hline CDG01-23 & 657 & 93 & -15.77 & 3.28 & 0.2 & 0.01239 \\
\hline CDG01-24 & 628 & 143 & -15.95 & 4.05 & 0.22 & 0.00933 \\
\hline CDG01-25 & 661 & 40 & -18.72 & 0.19 & 0.26 & 0.00456 \\
\hline CDG01-26 & 724 & 90 & -12.13 & 4.96 & 0.25 & 0.00472 \\
\hline CDG01-27 & 692 & 116 & -12.91 & 5.08 & 0.29 & 0.01205 \\
\hline CDG01-28 & 678 & 81 & -15.07 & 3.33 & 0.2 & 0.00615 \\
\hline CDG01-29 & 646 & 102 & -16.1 & 3.31 & 0.18 & 0.01455 \\
\hline CDG01-30 & 696 & 87 & -13.75 & 4.1 & 0.15 & 0.00681 \\
\hline \multicolumn{7}{|c|}{ Pulang Complex } \\
\hline PL1 & 710 & 469 & -6.86 & 9.72 & 0.68 & 0.031762 \\
\hline PL2 & 907 & 168 & -1.83 & 10.58 & 0.61 & 0.030845 \\
\hline PL3 & 762 & 117 & -9.39 & 5.94 & 0.65 & 0.031273 \\
\hline PL4 & 712 & - & & & 0.58 & 0.04695 \\
\hline PL5 & 739 & 38 & -14.75 & 1.12 & 0.6 & 0.026624 \\
\hline PL6 & 598 & - & & & 0.65 & 0.036933 \\
\hline PL7 & 658 & 18 & -22.12 & -4.15 & 0.62 & 0.02585 \\
\hline PL8 & 701 & 507 & -7.01 & 9.78 & 0.55 & 0.036627 \\
\hline PL9 & 768 & 77 & -10.67 & 4.51 & 0.72 & 0.055079 \\
\hline PL10 & 687 & - & & & 0.79 & 0.042448 \\
\hline PL11 & 744 & 72 & -12.14 & 3.61 & 0.67 & 0.036617 \\
\hline PL012 & 656 & 170 & -13.71 & 4.31 & 0.74 & 0.052305 \\
\hline PL013 & 701 & - & & & 0.72 & 0.055537 \\
\hline PL14 & 676 & 87 & -15.09 & 2.38 & 0.76 & 0.038616 \\
\hline PL15 & 681 & 1586 & -3.87 & 13.46 & 0.61 & 0.03808 \\
\hline
\end{tabular}


Table A1. Cont.

\begin{tabular}{|c|c|c|c|c|c|c|}
\hline Spot. No & $\mathrm{T}$ (Ti in Zircon) & $\mathrm{CeN} / \mathrm{CeN}^{*}$ & $\log f \mathrm{O}_{2}$ & $\delta F M Q$ & $\mathrm{EuN} / \mathrm{EuN}^{*}$ & $\mathrm{Ce} / \mathrm{Nd} / \mathrm{Y}$ \\
\hline \multicolumn{7}{|c|}{ Pulang Complex } \\
\hline PL16 & 692 & 262 & -10.02 & 7.02 & 0.65 & 0.045514 \\
\hline PL17 & 719 & 63 & -13.92 & 2.43 & 0.56 & 0.033955 \\
\hline PL18 & 735 & 189 & -8.95 & 7.02 & 0.53 & 0.050858 \\
\hline PL19 & 745 & 178 & -8.66 & 7.06 & 0.63 & 0.036346 \\
\hline PL20 & 560 & 71 & -23.48 & -2.43 & 0.62 & 0.057586 \\
\hline PL21 & 692 & 34 & -17.69 & -0.65 & 0.68 & 0.016862 \\
\hline PL22 & 679 & 50 & -16.97 & 0.41 & 0.72 & 0.035223 \\
\hline PL23 & 679 & 115 & -13.81 & 3.56 & 0.56 & 0.01685 \\
\hline PL024 & 703 & 181 & -10.79 & 5.96 & 0.54 & 0.180001 \\
\hline PL021 & 761 & 98 & -10.1 & 5.24 & 0.64 & 0.04412 \\
\hline PL022 & 685 & - & & & 0.59 & 0.033711 \\
\hline PL023 & 762 & 4 & -22.14 & -6.81 & 0.59 & 0.007535 \\
\hline PL024 & 719 & 241 & -8.84 & 7.5 & 0.54 & 0.039809 \\
\hline PL025 & 752 & 81 & -11.26 & 4.3 & 0.57 & 0.014062 \\
\hline PL026 & 692 & 118 & -13.03 & 4.01 & 0.59 & 0.043575 \\
\hline PL027 & 719 & 33 & -16.28 & 0.06 & 0.59 & 0.037696 \\
\hline PL028 & 762 & 184 & -7.7 & 7.63 & 0.62 & 0.031683 \\
\hline PL029 & 747 & 79 & -11.64 & 4.03 & 0.56 & 0.032735 \\
\hline PL-S210 & 630 & 28 & -22.03 & -3.27 & 0.53 & 0.013966 \\
\hline PL-S11 & 660 & 116 & -14.91 & 3 & 0.6 & 0.018867 \\
\hline PL-S12 & 730 & 214 & -8.71 & 7.36 & 0.48 & 0.041371 \\
\hline PL-S13 & 673 & 339 & -10.13 & 7.42 & 0.61 & 0.04972 \\
\hline PL-S14 & 666 & - & & & 0.65 & 0.041221 \\
\hline PL-S15 & 766 & 125 & -8.96 & 6.27 & 0.65 & 0.049746 \\
\hline PL-S16 & 686 & 85 & -14.56 & 2.62 & 0.64 & 0.061066 \\
\hline PL-S17 & 753 & - & & & 0.36 & 0.08668 \\
\hline PL-S18 & 706 & 198 & -10.31 & 6.37 & 0.48 & 0.053921 \\
\hline PL-S19 & 659 & 1792 & -4.71 & 13.23 & 0.63 & 0.045295 \\
\hline PL-S20 & 660 & 4 & -27.36 & -9.45 & 0.55 & 0.015036 \\
\hline PL-S21 & 680 & - & & & 0.67 & 0.039204 \\
\hline PL-S22 & 719 & 3 & -25.61 & -9.25 & 0.6 & 0.008073 \\
\hline PL-S23 & 676 & 141 & -13.28 & 4.19 & 0.66 & 0.03328 \\
\hline PL-S24 & 731 & 62 & -13.33 & 2.71 & 0.7 & 0.026698 \\
\hline PL031 & 731 & - & & & 0.66 & 0.032214 \\
\hline PL032 & 696 & 157 & -11.52 & 5.41 & 0.55 & 0.050453 \\
\hline PL033 & 724 & 102 & -11.61 & 4.6 & 0.51 & 0.025244 \\
\hline PL034 & 737 & - & & & 0.5 & 0.03888 \\
\hline PL035 & 692 & 41 & -16.81 & 0.22 & 0.5 & 0.037543 \\
\hline PL036 & 727 & 288 & -7.6 & 8.56 & 0.5 & 0.034121 \\
\hline PL037 & 764 & 277 & -5.9 & 9.39 & 0.64 & 0.03397 \\
\hline PL038 & 737 & 178 & -8.85 & 7.05 & 0.43 & 0.026517 \\
\hline PL039 & 739 & - & & & 0.5 & 0.053342 \\
\hline PL0310 & 735 & 154 & -9.52 & 6.44 & 0.54 & 0.04403 \\
\hline PL0311 & 702 & 81 & -13.69 & 3.09 & 0.49 & 0.031541 \\
\hline PL0312 & 697 & 308 & -8.93 & 7.97 & 0.48 & 0.045628 \\
\hline PL0313 & 662 & 60 & -17.11 & 0.73 & 0.46 & 0.062515 \\
\hline PL0314 & 668 & 90 & -15.26 & 2.44 & 0.52 & 0.006801 \\
\hline PL0315 & 722 & 152 & -10.21 & 6.05 & 0.47 & 0.036551 \\
\hline PL0316 & 699 & 228 & -9.98 & 6.89 & 0.55 & 0.035842 \\
\hline PL0317 & 765 & 23 & -15.15 & 0.1 & 0.51 & 0.01347 \\
\hline PL0318 & 709 & 44 & -15.61 & 1 & 0.41 & 0.025461 \\
\hline PL0319 & 710 & 254 & -8.94 & 7.63 & 0.51 & 0.033803 \\
\hline PL0320 & 702 & 153 & -11.27 & 5.5 & 0.54 & 0.023279 \\
\hline PL0321 & 691 & 45 & -16.45 & 0.61 & 0.54 & 0.042942 \\
\hline PL0322 & 731 & 83 & -12.08 & 3.98 & 0.56 & 0.032442 \\
\hline
\end{tabular}


Table A1. Cont.

\begin{tabular}{|c|c|c|c|c|c|c|}
\hline Spot. No & $\mathrm{T}$ (Ti in Zircon) & $\mathrm{CeN} / \mathrm{CeN}^{*}$ & $\log f \mathrm{O}_{2}$ & $\delta F M Q$ & $\mathrm{EuN} / \mathrm{EuN}^{*}$ & $\mathrm{Ce} / \mathrm{Nd} / \mathrm{Y}$ \\
\hline \multicolumn{7}{|c|}{ Lannitang Granite } \\
\hline LN7-2 & 789 & 112 & -8.14 & 7.28 & 0.77 & \\
\hline 1 & 827 & 87 & -7.37 & 7.14 & 0.67 & \\
\hline 2 & 856 & 135 & -4.51 & 9.38 & 0.72 & \\
\hline 3 & 1001 & 56 & -2.54 & 8.58 & 0.54 & \\
\hline 4 & 790 & 83 & -9.2 & 6.18 & 0.77 & \\
\hline 5 & 791 & 43 & -11.67 & 3.69 & 0.66 & \\
\hline 6 & 798 & 122 & -7.38 & 7.81 & 0.86 & \\
\hline 7 & 784 & 94 & -9 & 6.52 & 0.61 & \\
\hline 8 & 792 & 28 & -13.22 & 2.11 & 0.69 & \\
\hline 9 & 758 & 116 & -9.49 & 6.7 & 0.95 & \\
\hline LN1401 & 764 & 30 & -14.26 & 1.75 & 0.72 & \\
\hline 1 & 788 & 62 & -10.42 & 5.01 & 0.75 & \\
\hline 2 & 829 & 108 & -6.48 & 7.99 & 0.74 & \\
\hline 3 & 743 & 52 & -13.22 & 3.33 & 0.64 & \\
\hline 4 & 754 & 43 & -13.41 & 2.88 & 0.73 & \\
\hline 5 & 797 & 65 & -9.8 & 5.4 & 0.89 & \\
\hline 6 & 972 & 39 & -4.87 & 6.75 & 0.95 & \\
\hline 7 & 814 & 22 & -13.12 & 1.68 & 0.67 & \\
\hline 8 & 741 & 84 & -11.51 & 5.1 & 0.61 & \\
\hline 9 & 608 & 83 & -19.32 & 1.37 & 0.99 & \\
\hline
\end{tabular}

Notes: 1 Temperatures were calculated with a Ti-in-zircon thermometer [37]. 2 Oxygen fugacities ware calculated by the method proposed by [34]. $3 \mathrm{Eu}_{N} / \mathrm{Eu}_{\mathrm{N}}{ }^{*}=\mathrm{Eu}_{\mathrm{N}} / \mathrm{SQRT}\left(\mathrm{Sm}_{\mathrm{N}}{ }^{*} \mathrm{Gd}_{\mathrm{N}}\right)$, where subscript $\mathrm{N}$ refers to Chondrite normalized with the date of Sun and McDonough [48]. 4 Trace elements data from [5] for Pulang complex and Yu et al. [75] for Lannitang granite.

\section{References}

1. Deng, J.; Wang, Q.F.; Li, G.J.; Li, C.S.; Wang, C.M. Tethys tectonic evolution and its bearing on the distribution of important mineral deposits in the Sanjiang region, SW China. Gondwana Res. 2017, 26, 419-437. [CrossRef]

2. Roger, F.; Jolivet, M.; Malavieille, J. The tectonic evolution of the Songpan-Garzê (North Tibet) and adjacent areas from Proterozoic to Present: A synthesis. J. Asian Earth Sci. 2010, 39, 254-269. [CrossRef]

3. Wang, P.; Dong, G.C.; Santosh, M.; Li, X.F.; Dong, M.L. Triassic ore-bearing and barren porphyries in the Zhongdian arc of SW China: Implications for the subduction of the Paleo-Tethys Ocean. Int. Geol. Rev. 2017, 59, 1490-1505. [CrossRef]

4. Wang, P.; Dong, G.C.; Dong, M.L.; Li, Y.P.; Xu, Y.M.; Pan, Y.N.; Chen, W.; Wu, Z.C. Magma mixing of the Cuojiaoma batholith in the Yidun Arc: Evidence from mafic microgranular enclaves. Acta Petrol. Sin. 2017, 33, 2535-2547.

5. Meng, X.Y.; Mao, J.W.; Zhang, C.Q.; Zhang, D.Y.; Liu, H. Melt recharge, $f \mathrm{O}_{2}-\mathrm{T}$ conditions, and metal fertility of felsic magmas: Zircon trace element chemistry of $\mathrm{Cu}$-Au porphyries in the Sanjiang orogenic belt, southwest China. Miner. Depos. 2018, 53, 649-663. [CrossRef]

6. Wang, P.; Dong, G.C.; Zhao, G.C. Petrogenesis of the Pulang Porphyry Complex, Southwestern China: Implications for Porphyry Copper Metallogenesis and Subduction of the Paleo-Tethys Oceanic Lithosphere. Lithos 2018, 304-307, 280-297. [CrossRef]

7. Gao, X.; Yang, L.Q.; Evan, A.O. The lithospheric architecture of two subterranes in the eastern Yidun Terrane, East Tethys: Insights from Hf-Nd isotopic mapping. Gondwana Res. 2018, 62, 127-143. [CrossRef]

8. Zhang, X.; Wang, J. Zircon U-Pb dating and geochemistry of the Changdagou pluton in northwestern Sichuan Province. Acta Mineral. Sin. 2015, 35, 745-746. (In Chinese with English abstract)

9. Blevin, P.L. Redox and compositional parameters for interpreting the granitoid metallogeny of eastern Australia: Implications for gold-rich ore systems. Resour. Geol. 2004, 54, 241-252. [CrossRef]

10. Annen, C.; Blundy, J.; Sparks, R. The genesis of intermediate and silicic magmas in deep crustal hot zones. J. Petrol. 2006, 47, 505-539. [CrossRef] 
11. Loucks, R.R. Distinctive composition of copper-ore-forming arc mag-mas: Australian. J. Earth Sci. 2014, 61, 5-16.

12. Dilles, J.H.; Kent, A.J.; Wooden, J.L.; Tosdal, R.M.; Koleszar, A.; Lee, R.G.; Farmer, L.P. Zircon compositional evidence for sulfur-degassing from ore-forming arc magmas. Econ. Geol. 2015, 110, 241-251. [CrossRef]

13. Ding, T.; Ma, D.S.; Lu, J.J.; Zhang, R.Q. Apatite in granitoids related to polymetallic mineral deposits in southeastern Hunan Province, Shi-Hang zone, China: Implications for petrogenesis and metallogenesis. Ore Geol. Rev. 2015, 69, 104-117. [CrossRef]

14. Lu, Y.J.; Loucks, R.R.; Fiorentini, M.; Mccuaig, T.C.; Evans, N.J.; Yang, Z.M.; Hou, Z.Q.; Kirkland, C.L.; Parra-Avila, L.A.; Kobussen, A.; et al. Chapter 13 Zircon Compositions as a Pathfinder for Porphyry $\mathrm{Cu} \pm \mathrm{Mo}$ \pm Au Deposits. Econ. Geol. 2016, 19, 329-347.

15. Ballard, J.R.; Palin, M.J.; Campbell, I.H. Relative oxidation states of magmas inferred from Ce (IV)/Ce (III) in zircon: Application to porphyry copper deposits of northern Chile. Contrib. Mineral. Petrol. 2002, 144, 347-364. [CrossRef]

16. He, D.F.; Zhu, W.G.; Zhong, H.; Ren, T.; Bai, Z.J.; Fan, H.P. Zircon U-Pb geochronology and elemental and Sr-Nd-Hf isotopic geochemistry of the Daocheng granitic pluton from the Yidun Arc, SW China. J. Asian Earth Sci. 2013, 67-68, 1-17. [CrossRef]

17. He, M.; Zheng, H.; Clift, P.D. Zircon U-Pb geochronology and Hf isotope data from the Yangtze River sands: Implications for major magmatic events and crustal evolution in Central China. Chem. Geol. 2013, 360-361, 186-203. [CrossRef]

18. Guitreau, M.; Blichert-Toft, J.; Martin, H.; Mojzsis, S.J.; Albarède, F. Hafnium isotope evidence from Archean granitic rocks for deep-mantle origin of continental crust. Earth Planet. Sci. Lett. 2012, 337-338, 211-223. [CrossRef]

19. Li, W.C.; Zeng, P.S.; Hou, Z.Q.; Noel, C.W. The Pulang porphyry copper deposit and associated felsic intrusions in Yunnan Province, Southwest China. Econ. Geol. 2011, 106, 79-92.

20. Hu, R.Z.; Zhou, M.F. Multiple Mesozoic mineralization events in South China-An introduction to the thematic issue. Miner. Depos. 2012, 47, 579-588. [CrossRef]

21. Xiao, L.; He, Q.; Pirajno, F.; Ni, P.; Du, J.X.; Wei, Q.R. Possible correlation between a mantle plume and the evolution of Paleo-Tethys Jinshajiang Ocean: Evidence from a volcanic rifted margin in the Xiaru-Tuoding area, Yunnan, SW China. Lithos 2008, 100, 112-126. [CrossRef]

22. Song, H.; Zhang, C.J.; Li, Y.G.; Zhong, W.L.; Li, J.Q. Geochemical constraints of trace elements on ore-forming fluids in the copper polymetallic deposits in the middle Sanjiang Belts. Acta Petrol. Sin. 2016, 32, 804-814, (In Chinese with English abstract).

23. Peng, T.; Zhao, G.; Fan, W.; Peng, B.; Mao, Y. Zircon geochronology and Hf isotopes of Mesozoic intrusive rocks from the Yidun terrane, Eastern Tibetan Plateau: Petrogenesis and their bearings with Cu mineralization. J. Asian Earth Sci. 2014, 80, 18-33. [CrossRef]

24. Leng, C.B.; Zhang, X.C.; Hu, R.Z.; Wang, S.X.; Zhong, H.; Wang, W.Q.; Bi, X.W. Zircon U-Pb and molybdenite Re-Os geochronology and $\mathrm{Sr}-\mathrm{Nd}-\mathrm{Pb}-\mathrm{Hf}$ isotopic constraints on the genesis of the Xuejiping porphyry copper deposit in Zhongdian, Northwest Yunnan, China. J. Asian Earth Sci. 2012, 60, 31-48. [CrossRef]

25. Leng, C.B.; Huang, Q.Y.; Zhang, X.C.; Wang, S.C.; Zhong, H.; Hu, R.Z.; Bi, X.W.; Zhu, J.J.; Wang, X.S. Petrogenesis of the Late Triassic volcanic rocks in the Southern Yidun arc, SW China: Constraints from the geochronology, geochemistry and Sr-Nd-Pb-Hf isotopes. Lithos 2014, 190-191, 363-382. [CrossRef]

26. Zong, K.Q.; Klemd, R.; Yuan, Y.; He, Z.Y.; Guo, J.L.; Shi, X.L.; Liu, Y.S.; Hu, Z.C.; Zhang, Z.M. The assembly of Rodinia: The correlation of early Neoproterozoic (ca. $900 \mathrm{Ma}$ ) high-grade metamorphism and continental arc formation in the southern Beishan Orogen, southern Central Asian Orogenic Belt (CAOB). Precambrian Res. 2017, 290, 32-48. [CrossRef]

27. Hu, Z.C.; Zhang, W.; Liu, Y.S.; Gao, S.; Li, M.; Zong, K.Q.; Chen, H.H.; Hu, S.H. “Wave” signal smoothing and mercury removing device for laser ablation quadrupole and multiple collector ICP-MS analysis: Application to lead isotope analysis. Anal. Chem. 2015, 87, 1152-1157. [CrossRef]

28. Liu, Y.S.; Gao, S.; Hu, Z.C.; Gao, C.G.; Zong, K.Q.; Wang, D.B. Continental and oceanic crust recycling-induced melt-peridotite interactions in the Trans-North China Orogen: U-Pb dating, Hf isotopes and trace elements in zircons of mantle xenoliths. J. Petrol. 2010, 51, 537-571. [CrossRef] 
29. Liu, Y.S.; Hu, Z.C.; Gao, S.; Günther, D.; Xu, J.; Gao, C.G.; Chen, H.H. In situ analysis of major and trace elements of anhydrous minerals by LA-ICP-MS without applying an internal standard. Chem. Geol. 2008, 257, 34-43. [CrossRef]

30. Ludwig, K.R. ISOPLOT 3.00: A Geochronological Toolkit for Microsoft Excel; Berkeley Geochronology Center: Berkeley, CA, USA, 2003.

31. Woodhead, J.D.; Hergt, J.M.; Shelley, M.; Eggins, S.; Kemp, R. Zircon Hf-isotope analysis with an excimer laser, depth profiling, ablation of complex geometries, and concomitant age estimation. Chem. Geol. 2004, 209, 121-135. [CrossRef]

32. Fisher, C.M.; Vervoort, J.D.; Hanchar, J.M. Guidelines for reporting zircon Hf isotopic data by LA-MC-ICPMS and potential pitfalls in the interpretation of these data. Chem. Geol. 2014, 363, 125-133. [CrossRef]

33. Blichert-Toft, J.; Chauvel, C.; Albarede, F. Separation of Hf and Lu for high-precision isotope analysis of rock samples by magnetic sector-multiple collector ICP-MS. Contrib. Mineral. Petrol. 1997, 127, 248-260. [CrossRef]

34. Trail, D.; Watson, E.B.; Tailby, N.D. Ce and Eu anomalies in zircon as proxies for the oxidation state of magmas. Geochim. Cosmochim. Acta 2012, 97, 70-87. [CrossRef]

35. Smythe, D.J.; Brenan, J.M. Cerium oxidation state in silicate melts: Combined fO2, temperature and compositional effects. Geochim. Cosmochim. Acta 2015, 170, 173-187. [CrossRef]

36. Smythe, D.J.; Brenan, J.M. Magmatic oxygen fugacity estimated using zircon melt partitioning of cerium. Earth Planet. Sci. Lett. 2016, 453, 260-266. [CrossRef]

37. Ferry, J.M.; Watson, E.B. New thermodynamic models and revised calibrations for the Ti-in-zircon and Zr-in-rutile thermometers. Contrib. Mineral. Petrol. 2007, 154, 429-437. [CrossRef]

38. Qiu, J.T.; Yu, X.Q.; Santosh, M.; Zhang, D.H.; Chen, S.Q.; Li, P.J. Geochronology and magmatic oxygen fugacity of the Tongcun molybdenum deposit, northwest Zhejiang, SE China. Miner. Depos. 2013, 48, 545-556. [CrossRef]

39. Qiu, J.T.; Yu, X.Q.; Santosh, M.; Li, P.J.; Zhang, D.H.; Xiong, G.Q.; Zhang, B.Y. The Late Mesozoic tectonic evolution and magmatic history of west Zhejiang, SE China: Implications for regional metallogeny. Int. J. Earth Sci. 2014, 103, 713-735. [CrossRef]

40. Loader, M.A.; Wilkinson, J.J.; Armstrong, R.N. The effect of titanite crystallization on Eu and Ce anomalies in zircon and its implications for the assessment of porphyry Cu deposit fertility. Earth Planet. Sci. Lett. 2017, 472, 107-119. [CrossRef]

41. Thomas, R. Determination of water contents of granite melt inclusions by confocal laser Raman microprobe spectroscopy. Am. Mineral. 2000, 85, 868-872. [CrossRef]

42. Thomas, R.; Davidson, P. Water in granite and pegmatite-forming melts. Ore Geol. Rev. 2012, 46, 32-46. [CrossRef]

43. Williamson, B.J.; Herrington, R.J.; Morris, A. Porphyry copper enrichment linked to excess aluminum in plagioclase. Nat. Geosci. 2015, 9, 237-241.

44. Burnham, C.W. Water and magmas; A mixing model. Geochim. Cosmochim. Acta 1975, 39, 1077-1084. [CrossRef]

45. Liang, H.Y.; Sun, W.D.; Su, W.C.; Zartman, R.E. Porphyry copper-gold mineralization at Yulong, China, promoted by decreasing redox potential during magnetite alteration. Econ. Geol. 2009, 104, 587-596. [CrossRef]

46. Zhang, W.; Lentz, D.R.; Thorne, K.G.; McFarlane, C. Geochemical characteristics of biotite from felsic intrusive rocks around the Sisson Brook W-Mo-Cu deposit, west-central New Brunswick: An indicator of halogen and oxygen fugacity of magmatic systems. Ore Geol. Rev. 2016, 77, 82-96. [CrossRef]

47. Zhang, J.R.; Li, X.Y.; Ye, M. Research progress on Ce anomaly method for calculating oxygen fugacity of granite and applicability. Chin. Rare Earths 2018, 39, 20-27. (In Chinese with English abstract)

48. Belousova, E.A.; Griffin, W.L.; Reilly, S.Y. Igneous zircon: Trace element composition as an indicator of source rock type. Contrib. Mineral. Petrol. 2002, 143, 602-622. [CrossRef]

49. Hoskin, P.W.O.; Schaltegger, U. The composition of zircon and igneous and metamorphic petrogenesis. Rev. Miner. Geochem. 2003, 53, 27-62. [CrossRef]

50. Zeng, Y.F. The Geological Characteristics and Discussions on the Genesis of Porphyry Copper Deposit in Changdagou. Master's Thesis, Chengdu University of Technology, Chengdu, China, 2015; 62p. (In Chinese with English abstract) 
51. Blichert-Toft, J.; Albarede, F. The Lu-Hf isotope geochemistry of chondrites and the evolution of the mantle-crust system. Earth Planet. Sci. Lett. 1997, 148, 243-258. [CrossRef]

52. Griffin, W.L.; Pearson, N.J.; Belousova, E.; Jackson, S.E.; van Achterbergh, E.; O’Reilly, S.Y.; Shee, S.R. The $\mathrm{Hf}$ isotope composition of cratonic mantle: LAM-MC-ICPMS analysis of zircon megacrysts in kimberlites. Geochim. Cosmochim. Acta 2000, 64, 133-147. [CrossRef]

53. Li, S.R.; Yang, X.G.; Cheng, D.G.; Guo, P. Metallogenic regularity and prospecting potential of manganese deposits in Sichuan. Acta Geol. Sichuan 2012, 32, 21-25, (In Chinese with English abstract).

54. Chen, J.L.; Xu, J.F.; Ren, J.B.; Huang, X.X.; Wang, B.D. Geochronology and geochemical characteristics of late Triassic porphyritic rocks from the Zhongdian arc, eastern Tibet, and their tectonic and metallogenic implications. Gondwana Res. 2014, 26, 492-504. [CrossRef]

55. Ren, J.B.; Xu, J.F.; Chen, J.L. Zircon geochronology and geological implications of ore-bearing porphyries from Zhongdian arc. Acta Petrol. Sin. 2011, 27, 2591-2599, (In Chinese with English abstract).

56. Reid, A.J.; Wilson, C.J.L.; Liu, S. Structural evidence for the Permo-Triassic tectonic evolution of the Yidun Arc, eastern Tibetan Plateau. J. Struct. Geol. 2005, 27, 119-137. [CrossRef]

57. Reid, A.J.; Wilson, C.J.L.; Shun, L.; Pearson, N.; Belousova, E. Mesozoic plutons of the Yidun Arc, SW China: $\mathrm{U} / \mathrm{Pb}$ geochronology and $\mathrm{Hf}$ isotopic signature. Ore Geol. Rev. 2007, 31, 88-106. [CrossRef]

58. Shen, P.; Hattori, K.; Pan, H.; Jackson, S.; Seitmuratova, E. Oxidation condition and metal fertility of granitic magmas: Zircon trace-element data from porphyry $\mathrm{Cu}$ deposits in the Central Asian Orogenic Belt. Econ. Geol. 2015, 110, 1861-1878. [CrossRef]

59. Sun, W.D.; Liang, H.Y.; Ling, M.X.; Zhan, M.Z.; Ding, X.; Zhang, H.; Yang, X.Y.; Li, Y.L.; Ireland, T.R.; Wei, Q.R. The link between reduced porphyry copper deposits and oxidized magmas. Geochim. Cosmochim. Acta 2013, 103, 263-275. [CrossRef]

60. Sun, W.D.; Huang, R.F.; Li, H.; Hu, Y.B.; Zhang, C.C.; Sun, S.J.; Zhang, L.P.; Ding, X.; Li, C.Y.; Zartman, R.E. Porphyry deposits and oxidized magmas. Ore Geol. Rev. 2015, 65, 97-131. [CrossRef]

61. Jugo, P.; Candela, P.; Piccoli, P. Magmatic sulfides and Au: Cu ratios in porphyry deposits: An experimental study of copper and gold partitioning at $850^{\circ} \mathrm{C}, 100 \mathrm{MPa}$ in a haplogranitic melt-pyrrhotite-intermediate solid solution-gold metal assemblage, at gas saturation. Lithos 1999, 46, 573-589. [CrossRef]

62. Mungall, J.E. Roasting the mantle: Slab melting and the genesis of major $\mathrm{Au}$ and $\mathrm{Au}-$ rich $\mathrm{Cu}$ deposits. Geology 2002, 30, 915-918. [CrossRef]

63. Mungall, J.E.; Brenan, J.M. Partitioning of platinum-group elements and Au between sulfide liquid and basalt and the origins of mantle-crust fractionation of the chalcophile. Chem. Geol. 2014, 120, 223-253. [CrossRef]

64. Nicholls, J. A simple thermodynamic model for estimating the solubility of $\mathrm{H}_{2} \mathrm{O}$ in magmas. Contrib. Mineral. Petrol. 1980, 74, 211-220. [CrossRef]

65. Sillitoe, R.H. Porphyry copper systems. Econ. Geol. 2010, 105, 3-41. [CrossRef]

66. Wang, R.; Richards, J.P.; Hou, Z.-Q.; Yang, Z.-M.; Gou, Z.-B.; DuFrane, S.A. Increasing magmatic oxidation state from Paleocene to Miocene in the eastern Gangdese Belt, Tibet: Implication for collision-related porphyry Cu-Mo \pm Au mineralization. Econ. Geol. 2014, 109, 1943-1965. [CrossRef]

67. Wang, R.; Richards, J.P.; Hou, Z.; Yang, Z.; DuFrane, S.A. Increased Magmatic Water Content-The Key to Oligo-Miocene Porphyry Cu-Mo \pm Au Formation in the Eastern Gangdese Belt, Tibet. Econ. Geol. 2014, 109, 1315-1339. [CrossRef]

68. Wang, F.Y.; Ling, M.X.; Ding, X.; Hu, Y.H.; Zhou, J.B.; Yang, X.Y.; Liang, H.Y.; Fan, W.M.; Sun, W. Mesozoic large magmatic events and mineralization in SE China: Oblique subduction of the Pacific plate. Int. Geol. Rev. 2011, 53, 704-726. [CrossRef]

69. Yavuz, F. Evaluating micas in petrologic and metallogenic aspect: I-Definitions and structure of the computer program MICA+. Comput. Geosci. 2003, 29, 1203-1213. [CrossRef]

70. Yavuz, F. Evaluating micas in petrologic and metallogenic aspect: Part II-Applications using the computer program Mica+. Comput. Geosci. 2003, 29, 1215-1228. [CrossRef]

71. Richards, J.P. The oxidation state, and sulfur and $\mathrm{Cu}$ contents of arc magmas: Implications for metallogeny. Lithos 2015, 233, 27-45. [CrossRef]

72. Cai, H.M.; Zhang, H.F.; Xu, W.C.; Shi, Z.L.; Yuan, H.L. Petrogenesis of Indosinian volcanic rocks in Songpan-Garze fold belt of the northeastern Tibetan Plateau: New evidence for lithospheric delamination. China Earth Sci. 2010, 53, 1316-1328. [CrossRef] 
73. Hou, Z.Q.; Gao, Y.F.; Qu, X.M.; Rui, Z.Y.; Mo, X.X. Origin of adakitic intrusions generated during mid-Miocene east-west extension in southern Tibet. Earth Planet. Sci. Lett. 2004, 220, 139-155. [CrossRef]

74. Hou, Z.Q.; Zheng, Y.C.; Yang, Z.M.; Rui, Z.Y.; Zhao, Z.D.; Jiang, S.H.; Qu, X.M.; Sun, Q.Z. Contribution of mantle components within juvenile lower-crust to collisional zone porphyry Cu systems in Tibet. Miner. Depos. 2013, 48, 173-192. [CrossRef]

75. Yu, Y.F.; Fei, G.C.; Li, Y.G.; Long, X.R.; Tian, E.Y.; Liu, G.Q.; Lv, F.M.; Hua, K.Q. Oxygen Fugacity Characteristics and Metallogenic Significance of Porphyry Copper Deposit in Zhongdian Island. Chin. Rare Earths 2018, 39, 20-27. (In Chinese with English abstract)

(C) 2019 by the authors. Licensee MDPI, Basel, Switzerland. This article is an open access article distributed under the terms and conditions of the Creative Commons Attribution (CC BY) license (http://creativecommons.org/licenses/by/4.0/). 